\title{
Desarrollo regional y terciarización: los casos de Guanajuato y Querétaro, México
}

Jordy Micheli Thirión

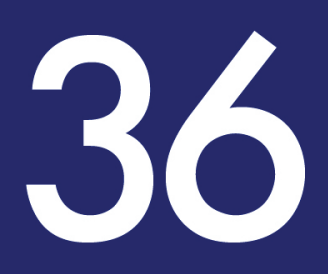

NOVIEMBRE/ DICIEMBRE 2016

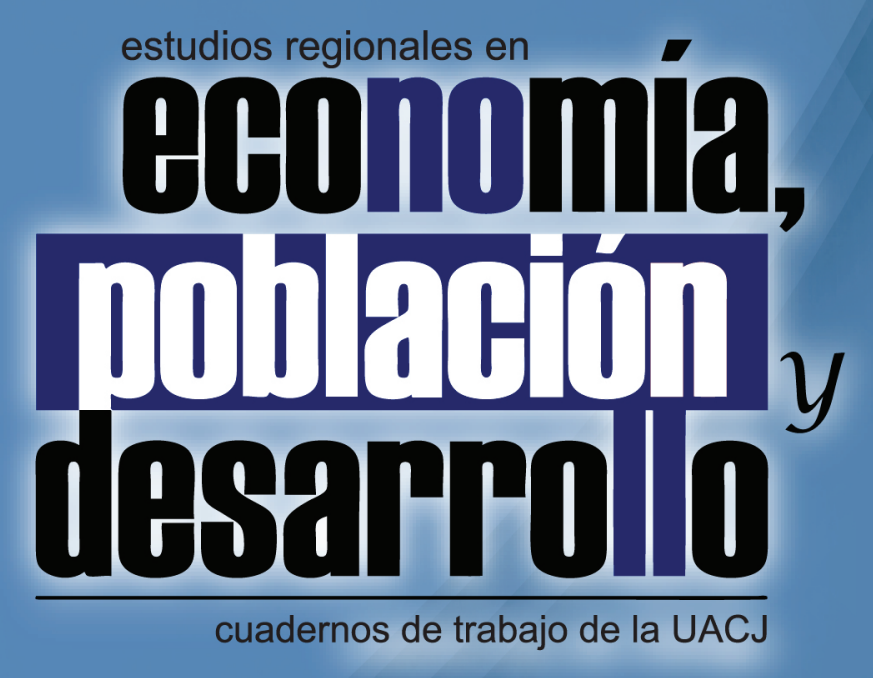




\section{Desarrollo regional y terciarización: los casos de Guanajuato y Querétaro, México}

Jordy Micheli Thirión 


\title{
UNIVERSIDAD AUTÓNOMA DE CIUDAD JUÁREZ \\ Instituto de Ciencias Sociales y Administración
}

\author{
Cuerpo Académico de Estudios Regionales en \\ Economía, Población y Desarrollo
}

Lic. Ricardo Duarte Jáquez

Rector

M.C. David Ramírez Perea

Secretario General

Mtro. Juan Ignacio Camargo Nassar

Director del Instituto de Ciencias

Sociales y Administración

Mtro. Ramón Chavira Chavira

Director General de Difusión

Cultura y Divulgación Científica

Dr. Luis Enrique Gutiérrez Casas

Coordinador General de

Investigación y Posgrado

\section{Dr. Jaime Alberto Arellano Quiroga \\ Coordinador del Cuerpo Académico de \\ Estudios Regionales en Economía, Población y Desarrollo}

Director y editor

Dr. Luis Enrique Gutiérrez Casas

Comité editorial

Sección internacional

Dra. Sofía Boza Martínez (Universidad de Chile, Chile)

Dra. Olga Biosca Artiñano (Glasgow Caledonian

University, Reino Unido)

Dra. Ángeles Sánchez Díez (Universidad Autónoma de

Madrid, España)

Dr. Thomas Fullerton Mankin (University of Texas at

E1 Paso, Estados Unidos)

Dr. Adrián Rodríguez Miranda (Universidad de la

República, Uruguay)

Sección local

(Universidad Autónoma de Ciudad Juárez)

Dra. Myrna Limas Hernández

Dra. Ikuho Kochi

Dr. Raúl Alberto Ponce Rodríguez

Dr. Isaac Leobardo Sánchez Juárez

Dr. Héctor Alonso Barajas Bustillos

Diseño de cubierta Abigail Bautista
Estudios Regionales en Economía, Población

y Desarrollo. Cuadernos de Trabajo de la UACJ

ISSN 2007-3739

Número 36. Noviembre/ Diciembre 2016

Desarrollo regional y terciarización: los casos de

Guanajuato y Querétaro, México

Jordy Micheli Thirión

Universidad Autónoma de Ciudad Juárez

Estudios Regionales en Economía, Población y Desarrollo. Cuadernos de Trabajo de la UACJ

Año 6, No. 36 noviembre - diciembre 2016, es una publicación bimestral editada por la Universidad Autónoma de Ciudad Juárez a través del Cuerpo Académico de Estudios Regionales en Economía, Población y Desarrollo del Instituto de Ciencias Sociales y Administración. Redacción: Avenida Universidad y H. Colegio Militar, Zona Chamizal s/n., C.P. 32300, Ciudad Juárez, Chihuahua, México. Teléfonos: (656) 688-38-00, ext. 3792. Correo electrónico: lgtz@uacj.mx.

Editor responsable: Luis Enrique Gutiérrez Casas. Reserva de derechos al uso exclusivo No. 04-2011-021713353900-102. ISSN 2007-3739, Impresa por Studio Los Dorados, calle Del Campanario, número 820-2, Santa Cecilia, C.P. 32350, Cd. Juárez, Chihuahua. Distribuidor: Subdirección de Gestión de Proyecto y Marketing Editorial. Ave. Plutarco Elías Calles 1210, Foviste Chamizal, C.P. 32310, Ciudad Juárez, Chihuahua. Este número se terminó de imprimir el 15 de octubre 2016 con un tiraje de 120 ejemplares.

Los ensayos publicados son responsabilidad exclusiva de sus autores. Se autoriza la reproducción total o parcial bajo condición de citar la fuente.

\section{Registrada en:}

Publicación afiliada a la Red Iberoamericana de Estudios del Desarrollo

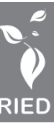

Universidad Autónoma de Ciudad Juárez

Ave Plutarco Elías Calles 1210

Foviste Chamizal, C.P. 32310

Ciudad Juárez, Chihuahua, México

www.uacj.mx

(C) Universidad Autónoma de Ciudad Juárez 
Estudios Regionales en Economía, Población y Desarrollo. Cuadernos de Trabajo de la UACJ, Universidad

Autónoma de Ciudad Juárez, número 36, noviembre-diciembre de 2016, ISSN 2007-3739, pp. 3-24, México.

\title{
Desarrollo regional y terciarización: los casos de Guanajuato y Querétaro, México
}

\author{
Jordy Micheli Thirión*
}

\begin{abstract}
Resumen
El trabajo tiene por objetivo construir y describir la configuración sectorial (manufactura y servicios) del desarrollo económico de Guanajuato y Querétaro en un período de 15 años. Nos basamos en el proceso de terciarización de la economía como característica contemporánea de los sistemas de producción nacionales y regionales. Aplicamos diversos instrumentos básicos de la Estadística aplicada a los procesos económicos: estructuras, indices y correlaciones, empleando información de los censos económicos de 1999 al 2014, del INEGI. Mostramos que la evolución sectorial de los dos estados se comporta de un modo convergente de forma tal que a lo largo de los 15 años se ha constituido una región homogénea estructuralmente, con un proceso de terciarización similar. La constitución de una región única en términos estructurales, que abarca a Guanajuato y Querétaro, es un fenómeno de la geografía económica que puede servir de base a estudios e interpretaciones del desarrollo regional y la terciarización en México.
\end{abstract}

Palabras clave: Tercerización, servicios, manufactura, Guanajuato, Querétaro.

\begin{abstract}
The aim of this work is to describe the sectoral configuration of the Mexican states of Guanajuato and Querétaro in terms of the evolution of manufacturing and services sectors in a 15 years period. I rely on the economy tertiarization as a contemporary characteristic of the national and regional productive systems. Three basic statistical instruments are applied for the analysis of economic development: structures: index numbers, and correlations, using the INEGI's national census from 1999 to 2014. The finding is that the sectoral evolution of both states converge, and as a result, in the period under study, a structurally homogenous region has emerged, sharing a similar tertiarization path. I suggest that the formation of an economically unified region in structural terms, can be considered a new phenomenon of the economic Geography in México that can serve as a basis for new analysis and interpretations of the regional development under a tertiarization perspective.
\end{abstract}

Key words:Tercerization, services, manufacture, Guanajuato, Querétaro.

JEL Classification: $R 10,014$

- Recibido en: Julio de 2016

- Aprobado en: Septiembre de 2016

\footnotetext{
* Jordy Micheli Thirión es profesor del Departamento de Economía de la Universidad Autónoma Metropolitana-Azcapotzalco. Correo electrónico: jordy.micheli@gmail.com.
} 


\section{$\rightarrow$ 1. Introduction.}

En el marco de la descentralización industrial que se produjo durante los años 80 del siglo pasado, en que diversas plantas y empresas de producción de manufacturas, especialmente del sector automotriz, comenzaron a abandonar la zona del Distrito Federal y sus cercanías, se configuró una importante capacidad productiva en algunos estados del norte del país y en la región central, principalmente en los estados de Guanajuato y Querétaro.

Estas dos entidades, en particular, se han caracterizado en los últimos tiempos por su dinamismo en sectores de la manufactura y los servicios: en el período de 2003 a 2013, mientras que el VACB ${ }^{1}$ manufacturero nacional creció a una tasa media de 1.98\% (a precios de 2008), en Guanajuato la manufactura creció al $2.42 \%$ y en Querétaro al 3.53\%. Igual circunstancia ocurrió con los servicios, que con una tasa media nacional de 3.37\%, fue superada por Guanajuato con $3.68 \%$ y por Querétaro con $5.48 \%$.

Por ello, en este artículo abordamos el caso de estos dos estados por su relevancia en el contexto de la industrialización nacional en su etapa contemporánea. Nos interesa una visión estructural a través de las estadísticas tradicionales sobre el desempeño de los sectores económicos (bienes y servicios desagregados) a lo largo de un período de tiempo. Este enfoque estructural de corte longitudinal permite trazar también un cuadro del desarrollo económico al incluir una visión de la terciarización de la economía regional y su coevolución con la manufactura² ${ }^{2}$ Este artículo se inscribe en la línea de trabajo del desarrollo regional basado en la terciarización en Micheli, 2014, y Micheli y Valle, 2016.

Con este enfoque observamos que se ha producido una senda del desarrollo económico similar en ambos estados, por lo cual convergen y se transforman en una región única desde el punto de vista de su desempeño sectorial de manufactura y servicios.

1 "El Valor Agregado censal Bruto (VACB) es el valor de la producción que se añade durante el proceso de trabajo, por la actividad creadora y de transformación del personal ocupado, el capital y la organización (factores de la producción), ejercida sobre los materiales que se consumen en la realización de la actividad económica. Aritméticamente, el VACB resulta de restar a la producción bruta total el consumo intermedio; se le llama bruto, porque no se le ha deducido el consumo de capital fijo.

2 Los servicios están presentes de modo continuo en el desarrollo y modos de consumo de los productos que son resultado de transformaciones de materiales, de modo que es sabido ya que existe una imbricación entre producción y consumo de bienes y de servicios. Esta es una característica estructural de la economía actual y que recibe diferentes nombres, nosotros la denominaremos de modo sintético terciarización de la economía, significando con ello tanto la dimensión cualitativa del proceso de coproducción bienes y servicios como la cuantitativa de la distribución tradicional de valor de la producción, empleo, etc. Nuestro interés es conocer las relaciones funcionales de determinados servicios avanzados con la producción manufacturera en los sistemas productivos territoriales, como un instrumento más de análisis del desarrollo local. 


\section{Los servicios avanzados en el desarrollo económico.}

En la estructura económica actual, los servicios ocupan ya un papel dominante en términos de producción, empleo y dinamismo innovador. Por tanto, para analizar las condiciones del desarrollo de países y regiones es necesario comprender las articulaciones entre los sectores de la manufactura, que han sido clásicamente los considerados artífices del desarrollo ${ }^{3}$ y los de los servicios, entendiendo la diversidad de éstos y su desigual importancia como ámbitos de trabajo en términos de remuneración, calidad del trabajo, productividad, etc. (Daniels; Bryson, 2002); (Pilat; Wölfl, 2005).

Una economía crecientemente diversificada, que incluye la producción de servicios está asociada a mayor productividad e ingresos, al nivel regional (Maillat; Bailly, 1989); (Hansen, 1990); (Greenfield, 1996). Reivindica Baró (2013, p. 38) la existencia de importantes cambios: “La metamorfosis de la industria, que ha tenido lugar en las últimas décadas, ha puesto de manifiesto cambios tanto en la naturaleza del «producto» - en la mayoría de casos, un «híbrido» de bienes (tangibles) y de prestaciones de servicios-, como en la (re)configuración de los procesos de generación de valor -en los que las fases de la cadena propiamente manufactureras ocupan un espacio cada vez menos relevante y, por el contrario, ganan en importancia las fases de creación, diseño y, ulteriormente, las fases de distribución, venta y post-venta del producto-, como, finalmente, en los profundos cambios en la geografía de esta «nueva» industria y en sus elementos potenciadores (activos logísticos, dotación de una base eficiente de servicios de apoyo a la fabricación, distribución y financiación de sus productos....)" (Baró, 2013)

En el conjunto de las economías terciarizadas, existe una clase de servicios, que en nuestro caso denominaremos como "avanzados" que sobresalen por su dinamismo y su vinculación con el desarrollo de un nuevo modelo productivo que combina manufactura y servicios, produciendo mayor valor agregado. Identificando a estos servicios, la Clasificación Internacional Industrial Unificada (CIIU) los agrupa en una División 8, la cual contiene a instituciones financieras, seguros, inmobiliarias y servicios a las empresas.

Utilizando los datos del Censo Económico de México de 2009, señalaremos como servicios avanzados a los servicios financieros y de seguros; corporativos; servicios profesionales, científicos y técnicos; servicios de apoyo a los negocios y manejo de desechos y servicios de re-

3 Recordemos, como un ejemplo clásico, la conceptualización de (Kaldor, 1966) que colocaba a la manufactura en el centro motriz del desarrollo mediante tres causas : 1) la tasa de crecimiento de una economía tiene una relación positiva con el sector manufacturero por su fuerte efecto multiplicador así como de encadenamientos y se fortalecerá la especialización conforme se incremente la expansión de la actividad manufacturera; 2) el incremento en la producción manufacturera llevará a un aumento de la productividad del trabajo en el mismo sector, ello provocado por los procesos de aprendizaje derivados de la división del trabajo; 3) la productividad en los sectores no manufactureros aumentará conforme la producción del sector manufacturero aumente. 
mediación; e información en medios masivos ${ }^{4}$. El peso de esta clase de servicios avanzados en la estructura del VACB nacional, ha crecido de 19.0 a $21.5 \%$, siendo el segundo sector específico con avances, detrás del acelerado crecimiento de la minería. Ello se muestra en el Cuadro 1.

Cuadro 1

Estructura \% del VACB nacional (1998-2013)

\begin{tabular}{|l|l|l|}
\hline & $\mathbf{1 9 9 8}$ & $\mathbf{2 0 1 3}$ \\
\hline Servicios & 51.4 & 47.8 \\
\hline Avanzados & 19.0 & 21.5 \\
\hline No avanzados & 32.4 & 26.3 \\
\hline Manufactura & 34.7 & 29.7 \\
\hline Minería & 8.2 & 16.3 \\
\hline Otros & 5.7 & 6.2 \\
\hline Total & 100.0 & 100.0 \\
\hline
\end{tabular}

Fuente: Elaboración propia con datos de los censos económicos 1999, 2004, 2009 y 2014, INEGI.

\section{La producción y empleo de servicios avanzados: comparación con la manufactura.}

Para contextualizar el significado del crecimiento que ha experimentado el sector de servicios avanzados, cabe señalar que su producción ha crecido a un ritmo tal que se ha ido acercando en valor a la producción de las manufacturas. En la Gráfica 1 se observa que la brecha de valor existente entre manufactura y servicios avanzados se redujo en $59 \%$ desde 2003 hasta 2013.

En cuanto al empleo, las diferencias entre manufacturas y servicios avanzados son más evidentes, y la brecha entre ambos sectores solo se ha reducido $17 \%$ de 2003 a 2013, tal se ilustra en la Gráfica 2.

El sector de servicios avanzados tiene una productividad laboral (Valor de la producción entre persona ocupada, anual) que se ha diferenciado cada vez más de la manufactura, la cual se ha mantenido prácticamente estancada. La brecha de productividad a favor de los servicios avanzados ha crecido en 67 \%, en el periodo 2003-2013. Ello se muestra en la Gráfica 3

4 Esta gran agrupación de servicios, contiene a la producción de software, lo cual es una razón importante para considerarla como servicios "avanzado" en los términos que nos interesan. 
Gráfica 1

Evolución de la manufactura y los servicios avanzados (PIB, millones de pesos a precios 2008)

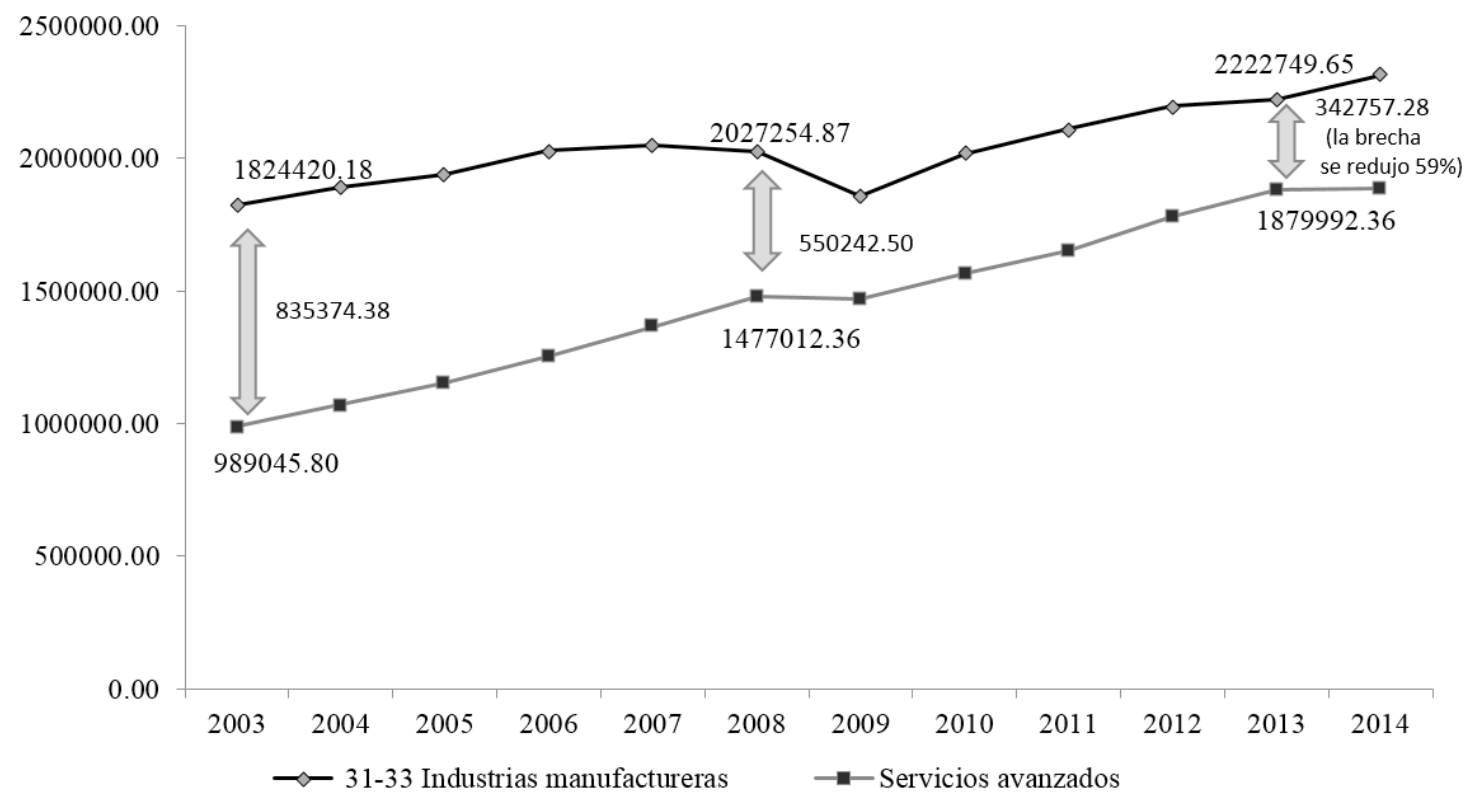

Fuente: Elaboración propia con datos del BIE, INEGI.

Gráfica 2

Evolución de la manufactura y los servicios avanzados (personal ocupado)

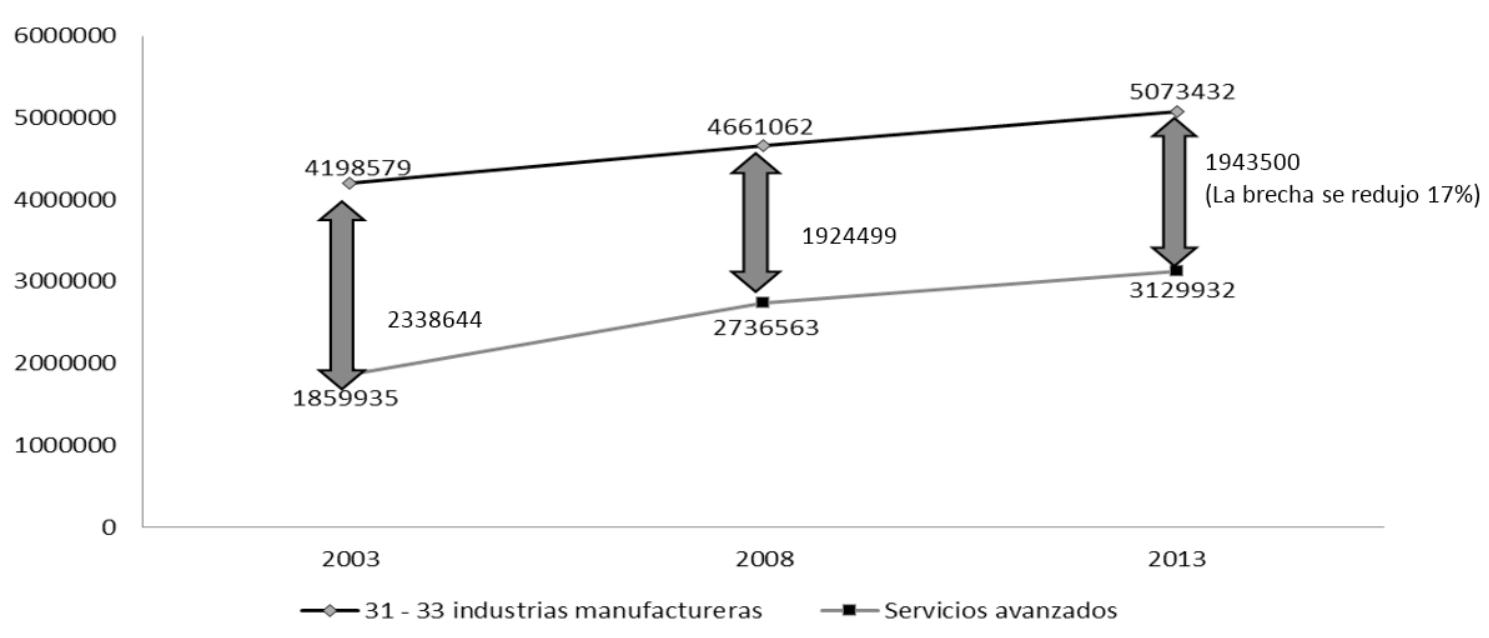

Fuente: Elaboración propia con datos del Censo Económico, INEGI. 
Gráfica 3

Productividad (miles de pesos a precios 2008)

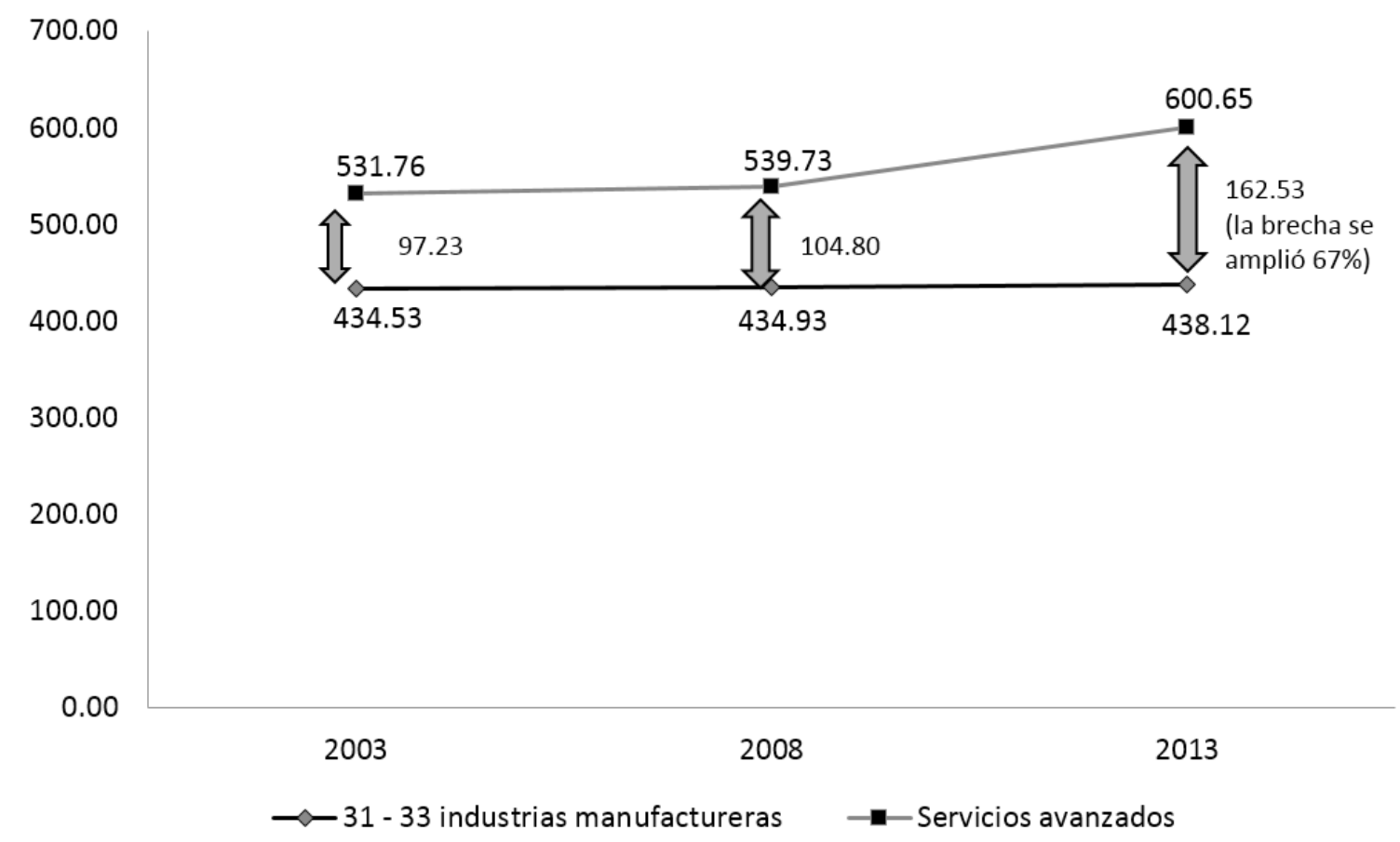

Fuente: Elaboración propia con datos del Censo Económico y el BIE, INEGI.

Para evaluar la sensibilidad que tienen los servicios frente a la manufactura, hemos calculado la correlación entre su valor de producción y un incremento de $\$ 1000$ pesos en el valor de la producción de manufactura (ver Anexo 1). La evolución de este dato la mostramos en el Cuadro 2, el cual ilustra que en la economía mexicana se ha producido un crecimiento mayor de la sensibilidad de servicios avanzados que del resto de servicios.

Cuadro 2

Producción adicional en servicios ante un incremento de \$1000 en manufactura (1998-2013)

\begin{tabular}{|l|l|l|l|l|}
\hline SERVICIOS & $\mathbf{1 9 9 8}$ & $\mathbf{2 0 0 3}$ & $\mathbf{2 0 0 8}$ & $\mathbf{2 0 1 3}$ \\
\hline Avanzados & 25 & 43 & 47 & 66 \\
\hline Resto de los servicios & 178 & 207 & 150 & 170 \\
\hline $\begin{array}{l}\text { Avanzados/Resto de los } \\
\text { servicios }\end{array}$ & 0.14 & 0.20 & 0.31 & 0.39 \\
\hline
\end{tabular}

Fuente: Elaboración propia con datos de los censos económicos 1999, 2004, 2009 y 2014, INEGI. 
En el marco de la preocupación por conocer las relaciones entre sectores de manufactura y de servicios, el índice que hemos elaborado tiene la utilidad de servir de dato de comparación entre regiones entre períodos, para dimensionar la coevolución estadística entre manufactura y servicios, especialmente los avanzados, que son los reconocidos como integrantes de una capacidad productiva regional de alta productividad y generadora de una senda moderna de desarrollo. Bajo este enfoque denominamos al índice como coevolución estadística entre manufactura y servicios en el análisis sectorial que abordamos.

\section{- 4. La visión mesoeconómica del desarrollo local.}

La desconcentración geográfica de la industrialización ha sido motivo de estudios académicos, especialmente desde una óptica mesoeconómica, con un fuerte componente de estudio de campo 5 . Los clusters y el escalamiento/aprendizaje son herramientas analíticas importantes para la investigación de los procesos locales de industrialización ligada a la inversión extranjera y la exportación, en un ámbito regional que abarca tanto a estados de la frontera norte como a los de la región del Bajío. Existe igualmente un interés por observar la relación de la industrialización con el capital social e institucional regional, y en particular en intentar mostrar cómo el desarrollo de mejoras y/o de innovaciones puntuales e incrementales, tanto en procesos como en productos, es el resultado de estrategias empresariales y de vínculos de las plantas productivas con el entramado de capacidades locales, especialmente del capital intangible (educación, conocimiento, confianza, ) y de las instituciones que las crean.

La literatura sobre actores, procesos y sistemas regionales de producción manufacturera ha creado una intersección entre las visiones de cadenas de valor internacionales y la construcción de un capital social regional. Ha producido importantes y variados análisis sobre realidades en que la producción tiene como origen la inversión extranjera y como destino el mercado estadunidense, básicamente. Así ha sido analizada la frontera norte y estados de dinamismo manufacturero como Guanajuato y Querétaro. De modo puntual presentamos algunas aportaciones significativas que ilustran el ámbito explicativo que se ha construido sobre las regiones.

5 En el conjunto de trabajos de esta corriente de estudios que unen al territorio con la industrialización desde una perspectiva mesoeconómica, caben mencionar, entre otros, a (Casalet, 2000) y (Casas, 2001) que tienen un papel importante por sus primeros estudios sobre el capital social regional; (Carrillo; Barajas, 2007), (Lara, 2007), (Dutrenit, 2009), (Hualde, 2010) y (Contreras; Isiordia, 2010), quienes analizan sectores y regiones, con un énfasis importante en procesos de aprendizaje y escalamiento. Recientemente ha adquirido importancia la búsqueda de procesos de innovación en la escala regional: (Villavicencio; López, 2009), (Rózga, 2010), (Bracamontes; Contreras, 2011), (Villavicencio, 2011). En general, el común denominador de estas aportaciones es el abandonar el enfoque de una división internacional del trabajo estática, con ventajas laborales basadas en trabajo barato y apto para el aprendizaje; a otro en la cual las preguntas son acerca del modo en que se crean oportunidades locales al estar implicadas en alguna parte de la cadena de valor global. 
Estrada analiza la composición tecnológica y el desempeño de la industria tradicional y de gran escala en Guanajuato y echa de menos "una integración más activa con los servicios modernos al productor: Se requieren servicios más elaborados de capacitación, mercadotecnia e I+D como se muestra en la débil demanda detectada en otros sectores productivos más modernos. Aunque es cierto que estos sectores dedican escasos recursos a estas actividades; esto es, no se tiene un suficiente desarrollo de estas fuentes internas de ventaja competitiva. En estas dimensiones es que Guanajuato presenta una gran desventaja respecto a México" (Estrada, 2006) (p. 857).

Escalante y Catalán, señalan que "La industria de manufacturas en Guanajuato tiene una estructura heterogé $\neg n e a$, cuyas actividades están orientadas a distintos mercados. Así, por ejemplo, la industria de autopartes se concentra (72\%) en la fabricación de partes de sis $\neg$ temas de transmisión, con un gran dinamismo y una fuerte orientación al mer $\neg$ cado externo, concentrada en los principales centros urbanos del estado.” (Escalante; Catalán, 2011) (p.109)

Álvarez analiza las capacidades industriales endógenas del estado de Guanajuato y las contrapone con las exógenas, alentadas por la política de apoyo a empresas multinacionales, como la General Motors: “. En el caso de Silao, a quince años de apertura de General Motors, su impacto territorial pareciera endeble. Pues el territorio se ve envuelto en procesos holísticos conectados a dinámicas económicas y flujos sociales interregionales, ligados por un lado, a procesos de migración laboral, y por otro a tasas de desempleo preocupantes en el municipio. Aproximadamente el diez por ciento de la plantilla laboral de General Motors se compone de trabajadores local" (Álvarez, 2013) (p. 9)

Adriana Martínez ha generado una rica literatura sobre el proceso de industrialización del estado, observando a los actores empresa, gobierno y entidades de investigación, para explicar la dinámica de sectores como el calzado y la industria automotriz, así como el sistema local de innovación (Martínez, 2009) (Martínez, 2012) (Martínez, 2015).

Para el estado de Querétaro, trabajos relevantes recientes en la óptica d la industrialización local son los que han fijado su atención en las empresas aeronáuticas (Casalet, 2013) (Villavicencio, 2013).

\section{$\Rightarrow$ 5. El enfoque sectorial: manufactura y servicios en los estados de Guanajuato y Querétaro.}

\subsection{Participación en la producción nacional y estructura interna.}

El estudio abarca el periodo de 1998 a 2013, es decir, quince años. En ese lapso, los dos estados han ido perdiendo peso como productores de manufactura a nivel nacional ${ }^{6}$, pero han ganado peso en tanto productores de servicios, tanto de los no avanzados como de los avanzados. En todos los casos, la participación de Guanajuato es del doble que la de Querétaro. Ello se muestra en Gráfica 4. 
En concordancia con ese comportamiento a nivel nacional, su estructura interna sectorial se caracteriza por tener un fuerte componente de la manufactura: alrededor de $50 \%$, si bien con una clara tendencia a la baja. En sentido opuesto, los servicios, especialmente los avanzados tienden a crecer. En este punto, es fácil distinguir ya la convergencia de la configuración sectorial en ambos estados.

Gráfica 4

Participación nacional de Guanajuato y Querétaro en manufactura y servicios (1998-2013)

\begin{tabular}{|l|l|l|l|l|}
\hline SERVICIOS & $\mathbf{1 9 9 8}$ & $\mathbf{2 0 0 3}$ & $\mathbf{2 0 0 8}$ & $\mathbf{2 0 1 3}$ \\
\hline Avanzados & 25 & 43 & 47 & 66 \\
\hline Resto de los servicios & 178 & 207 & 150 & 170 \\
\hline $\begin{array}{l}\text { Avanzados/Resto de los } \\
\text { servicios }\end{array}$ & 0.14 & 0.20 & 0.31 & 0.39 \\
\hline
\end{tabular}

Fuente: Elaboración propia con datos de los censos económicos 1999, 2004, 2009 y 2014, INEGI.

Gráfica 5

Participación de manufactura y servicios en la producción de Guanajuato y Querétaro (1998-2013)

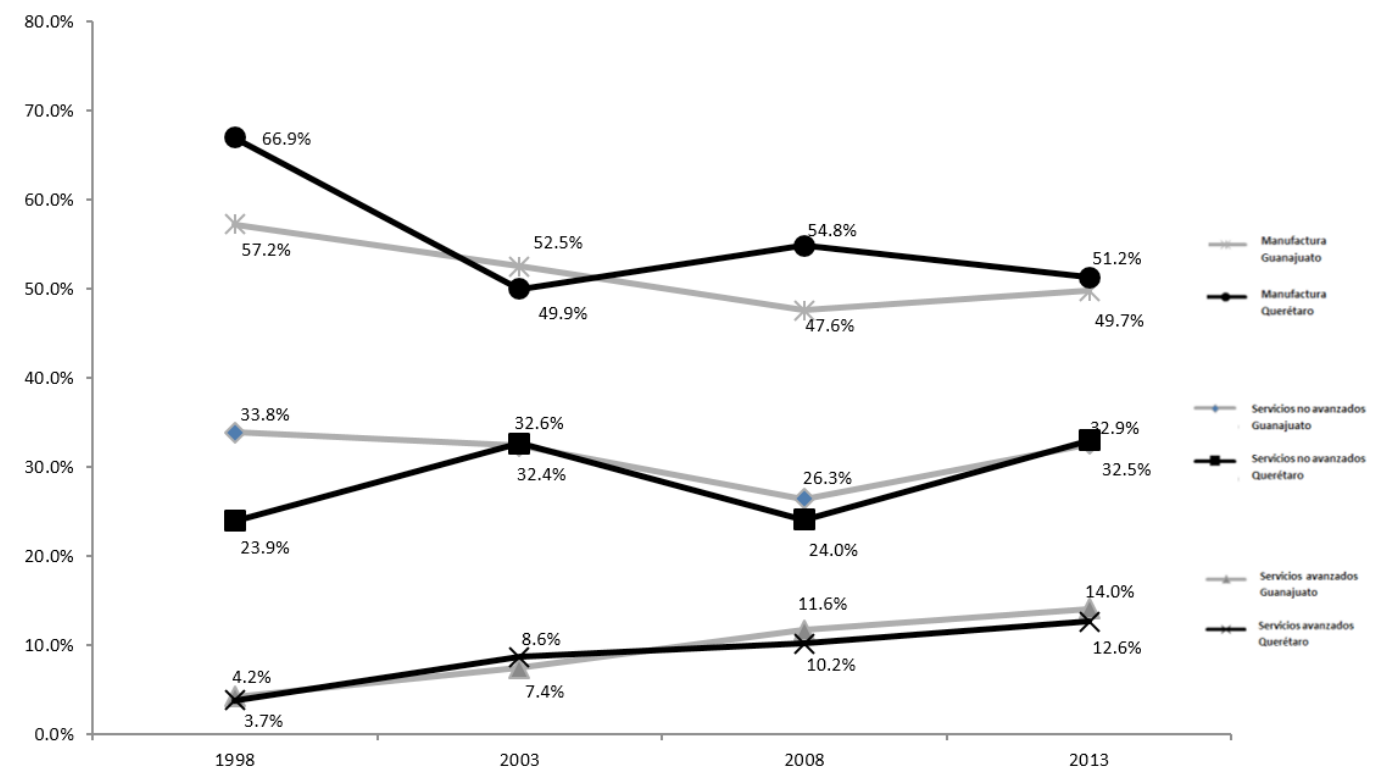

Fuente: Elaboración propia con datos de los censos económicos 1999, 2004, 2009 y 2014, INEGI.

6 Con fines comparativos, el Estado de México pasó de $16.9 \%$ a $11.0 \%$ en la manufactura nacional en el mismo período, en tanto que Puebla pasó de $4.2 \%$ a $10.8 \%$, representando ambos casos los de mayor variación a nivel nacional. El DF pasó de $10.4 \%$ a $6.2 \%$ y el estado de Nuevo León se mantuvo estable en $9.8 \%$. En ese sentido, nuestras dos entidades tienen poca pérdida de representatividad manufacturera, a nivel nacional. 


\subsection{Especialización y concentración manufactureras.}

Bajo la óptica que nos interesa, es relevante conocer las pautas de diversificación o concentración de la estructura manufacturera. Para ello utilizamos un par de índices comúnmente empleados.

El primero de ellos es el Índice de Especialización (ver Anexo 1) que permite conocer la importancia relativa de un sector determinado dentro de la estructura regional, comparándola con la importancia del mismo sector en la estructura nacional. Se forma así un índice que revela la especialización relativa de la región en el sector determinado. Como nuestra mirada recorre el período de 1998 a 2013, podemos observar si ha habido alguna variación de la especialización. Los sectores que siempre aparecen en esta observación de 15 años, nos permiten sugerir que constituyen la especialización "tradicional" del estado.

Como se ve en el cuadro 3, en ambos estados hay un grupo de sectores de la manufactura en que existe de modo persistente una especialización, y únicamente hay un nuevo sector de especialización, el mismo en cada estado: Industria del plástico y del hule.

El segundo indicador es el conocido Índice de Herfindhal e Hirschman, IHH, del cual elaboramos una serie para el mismo período 1998-2013 para la manufactura. Por tanto, queremos apoyar este primer resultado sobre la especialización relativa de la manufactura con una mirada hacia lo que ha ocurrido dentro de cada estado en el período analizado. En este índice, el valor cercano a 1000 denota una estructura productiva más diversificada, y en sentido inverso, el aumento de valor del índice denota una estructura concentrada ${ }^{7}$ (ver Anexo 1).

Los datos del IHH en estos dos estados denotan que en el último año de referencia, existe en ellos un nivel de diversificación idéntico en la manufactura, con la diferencia entre ambos estados en su recorrido, puesto que Guanajuato ha tendido hacia una desconcentración en tanto que Querétaro se ha mantenido estable. De nueva cuenta, el dato relevante es la convergencia. Ello se muestra en la Gráfica 6.

7 Cabe decir que el índice de especialización y el IHH tienen semejanzas pero no miden lo mismo: El primero relaciona valores internos y externos a la región estudiada, y el segundo refleja únicamente lo que sucede dentro del territorio. Estamos observando un período de 15 años y ello nos permite visualizar tendencias y por tanto un panorama amplio de evolución Por ejemplo, un estado puede arrojar tendencia hacia el alza del índice de especialización "Y" pero su IHH mantenerse constante, con lo cual se manifiesta que la producción de "Y" dentro del estado no está polarizando el aparato manufacturero. 
Cuadro 3

Especialización manufacturera de Guanajuato y Querétaro (1998-2013)

\begin{tabular}{|c|c|c|c|c|c|}
\hline \multicolumn{2}{|l|}{ GUANAJUATO } & \multirow{2}{*}{$\begin{array}{l}1998 \\
8,7\end{array}$} & \multirow{2}{*}{$\begin{array}{l}2003 \\
11,0\end{array}$} & \multirow{2}{*}{\begin{tabular}{r|}
2008 \\
12,9
\end{tabular}} & \multirow{2}{*}{$\begin{array}{l}2013 \\
12,0\end{array}$} \\
\hline $\begin{array}{l}\text { ESPECIALIZACION } \\
\text { TRADICIONAL }\end{array}$ & $\begin{array}{l}\text { Curtido y acabado de } \\
\text { cuero y piel, y } \\
\text { fabricación de productos } \\
\text { de cuero, piel y } \\
\text { materiales sucedáneos }\end{array}$ & & & & \\
\hline & $\begin{array}{l}\text { Fabricación de } \\
\text { productos derivados del } \\
\text { petróleo y del carbón }\end{array}$ & 4,6 & 2,3 & 7,2 & 2,0 \\
\hline & $\begin{array}{l}\text { Fabricación de equipo } \\
\text { de transporte }\end{array}$ & 3,1 & 2,1 & 1,2 & 1,0 \\
\hline $\begin{array}{l}\text { ESPECIALIZACION } \\
\text { RECIENTE }\end{array}$ & $\begin{array}{l}\text { Industria del plástico y } \\
\text { del hule }\end{array}$ & 0,5 & 0,8 & 1,1 & 1,6 \\
\hline \multicolumn{2}{|l|}{ QUERÉTARO } & 1998 & 2003 & 2008 & 2013 \\
\hline \multirow{7}{*}{$\begin{array}{l}\text { ESPECIALIZACION } \\
\text { TRADICIONAL }\end{array}$} & Industria del papel & 2,4 & 5,1 & 2,2 & 4,0 \\
\hline & $\begin{array}{l}\text { Impresión e industrias } \\
\text { conexas }\end{array}$ & 1,0 & 2,4 & 2,3 & 1,7 \\
\hline & $\begin{array}{l}\text { Fabricación } \\
\text { productos metálicos }\end{array}$ & 0,7 & 1,1 & 1,1 & 1,5 \\
\hline & $\begin{array}{l}\text { Fabricación de } \\
\text { maquinaria y equipo }\end{array}$ & 1,7 & 1,4 & 1,8 & 2,5 \\
\hline & $\begin{array}{l}\text { Fabricación de } \\
\text { accesorios, aparatos } \\
\text { eléctricos y equipo de } \\
\text { generación de energía } \\
\text { eléctrica }\end{array}$ & 1.5 & 2,2 & 1,5 & 1,9 \\
\hline & $\begin{array}{l}\text { Fabricación } \\
\text { productos metálicos }\end{array}$ & 0,7 & 1,1 & 1,1 & 1,5 \\
\hline & Industria alimentaria & 1,2 & 0,7 & 1,4 & 1,2 \\
\hline $\begin{array}{l}\text { ESPECIALIZACION } \\
\text { RECIENTE }\end{array}$ & $\begin{array}{l}\text { Industria del plástico y } \\
\text { del hule }\end{array}$ & 0,5 & 0,9 & 1,5 & 1,8 \\
\hline
\end{tabular}

Fuente: Elaboración propia con datos de los censos económicos 1999, 2004, 2009 y 2014, INEGI. 
Gráfica 6

\section{El índice de de Herfindhal e Hirschman de la manufactura en Guanajuato y Querétaro (1998-2013)}

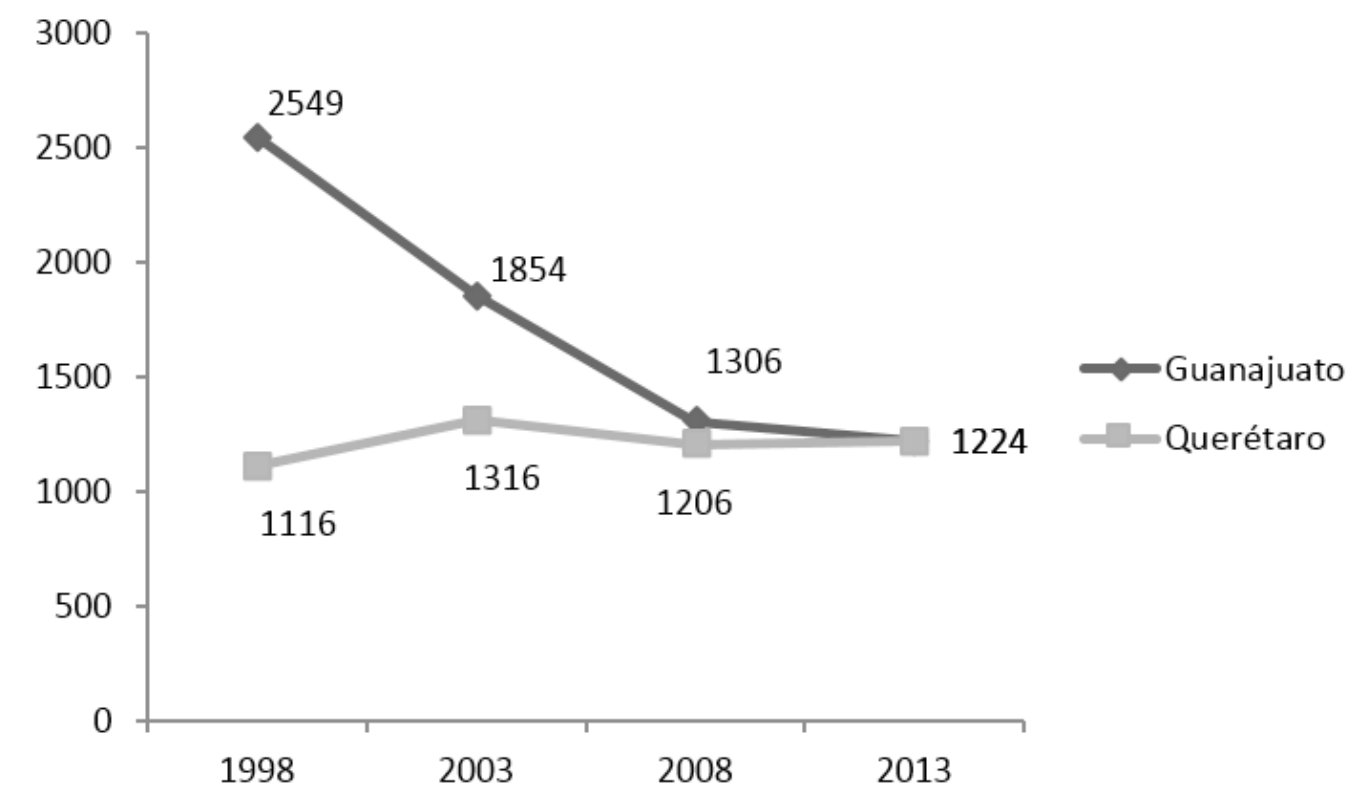

Fuente: Elaboración propia con datos de los censos económicos 1999, 2004, 2009 y 2014, INEGI.

\subsection{Sensibilidad de los servicios avanzados a la manufactura.}

¿Cómo han interactuado los dos sectores: manufactura y servicios avanzados? Empleando el índice de sensibilidad de los servicios avanzados a la manufactura, vemos que en ambos estados ha existido una tendencia ascendente que los ha colocado por encima del valor promedio nacional (mismo que se puede observar en el cuadro 2). Ello se muestra en la Gráfica 7. 


\section{Sensibilidad de los servicios avanzados a la manufactura en Guanajuato y Querétaro (1998-2013)}

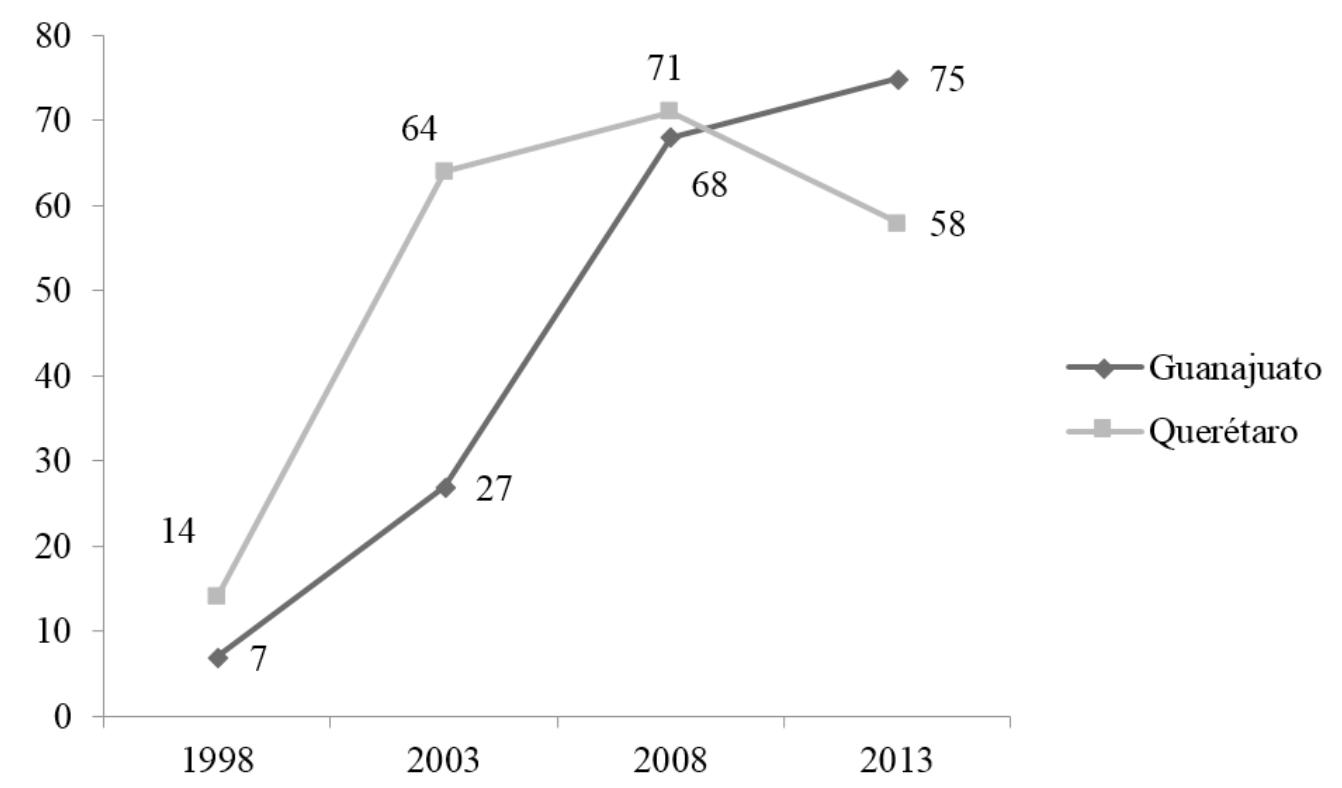

Fuente: Elaboración propia con datos de los censos económicos 1999, 2004, 2009 y 2014, INEGI.

\subsection{Productividad y remuneraciones de los sectores manufactura y servicios.}

En cada una de las tres gráficas siguientes se muestra la evolución de productividad y remuneraciones en VACB mensual por persona ocupada, a precios de 2008, por cada uno de los tres sectores que hemos contemplado, en ambos estados. Se observa que Querétaro tiene productividades y remuneraciones más altas que Guanajuato y que tendencialmente vuelve a manifestarse una convergencia entre ambas entidades. Las productividades de la manufactura van en descenso, las de servicios avanzados suben y son más elevadas que las de la manufactura y las del resto de servicios están prácticamente estables y claramente más bajas que las de los otros dos sectores. Las remuneraciones de la manufactura son estables mas no así las de los servicios, que son descendentes. 
Gráfica 8

Productividad y remuneraciones en la manufactura en

Guanajuato y Querétaro (1998-2013)

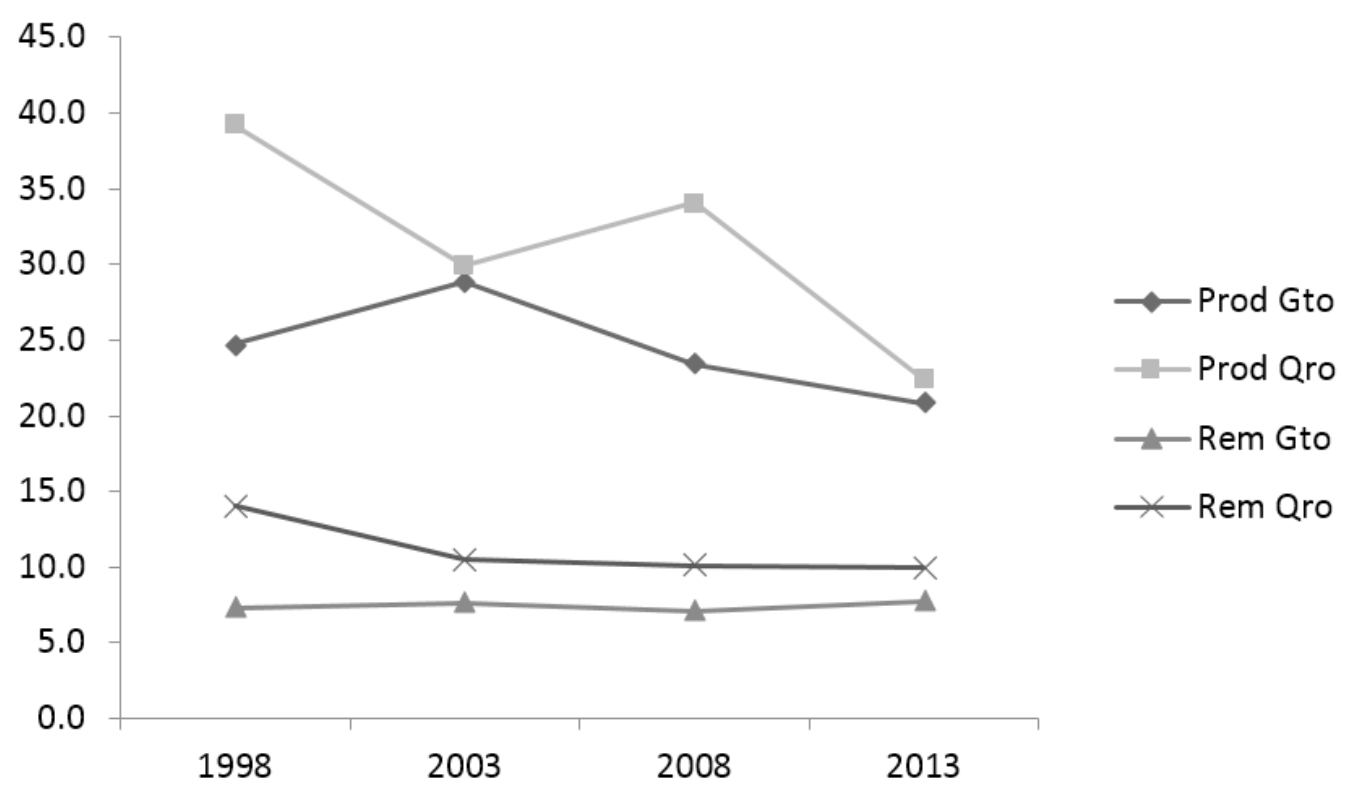

Fuente: Elaboración propia con datos de los censos económicos 1999, 2004, 2009 y 2014, INEGI.

Gráfica 9

Productividad y remuneraciones en los servicios avanzados en Guanajuato y Querétaro (1998-2013)

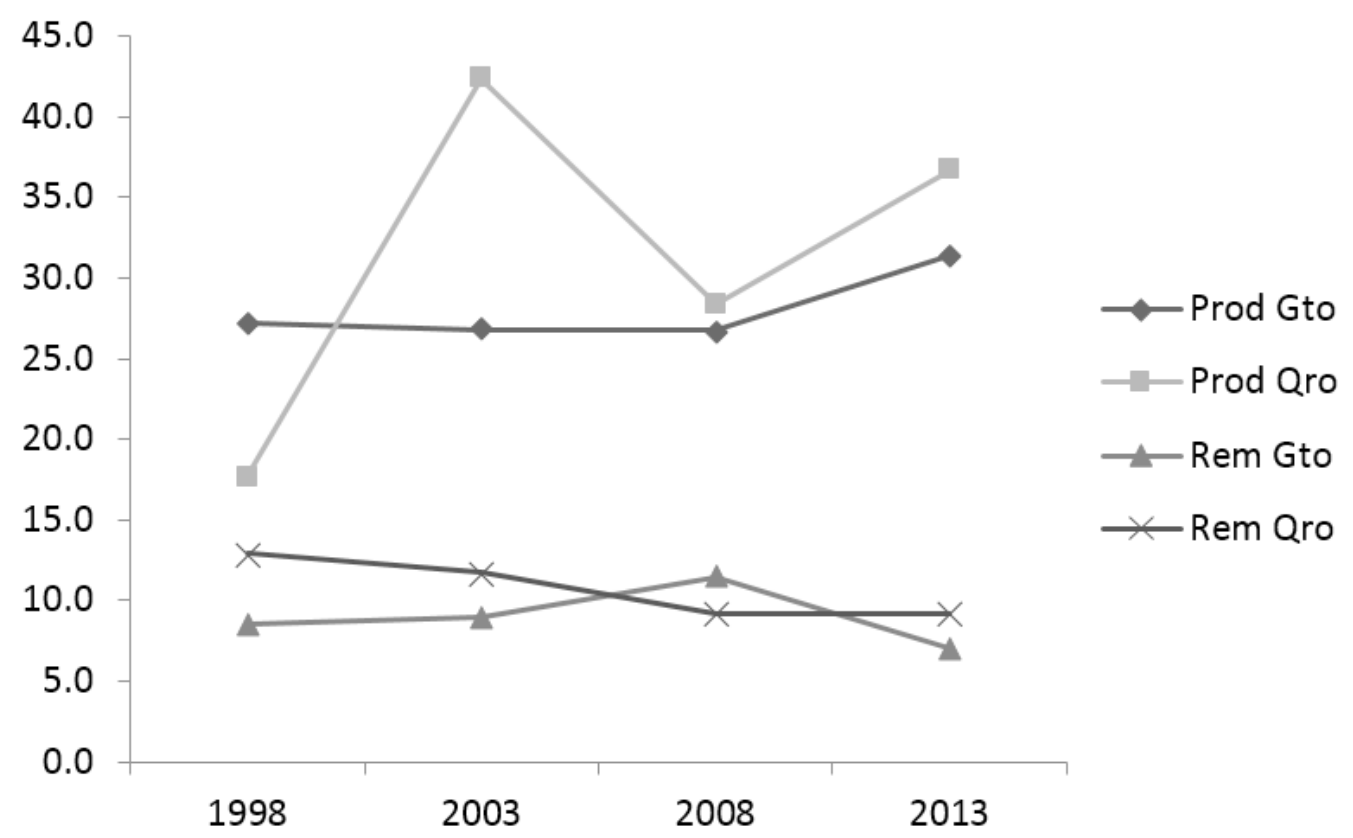

Fuente: Elaboración propia con datos de los censos económicos 1999, 2004, 2009 y 2014, INEGI. 
Gráfica 10

\section{Productividad y remuneraciones en los servicios no avanzados en Guanajuato y Querétaro (1998-2013)}

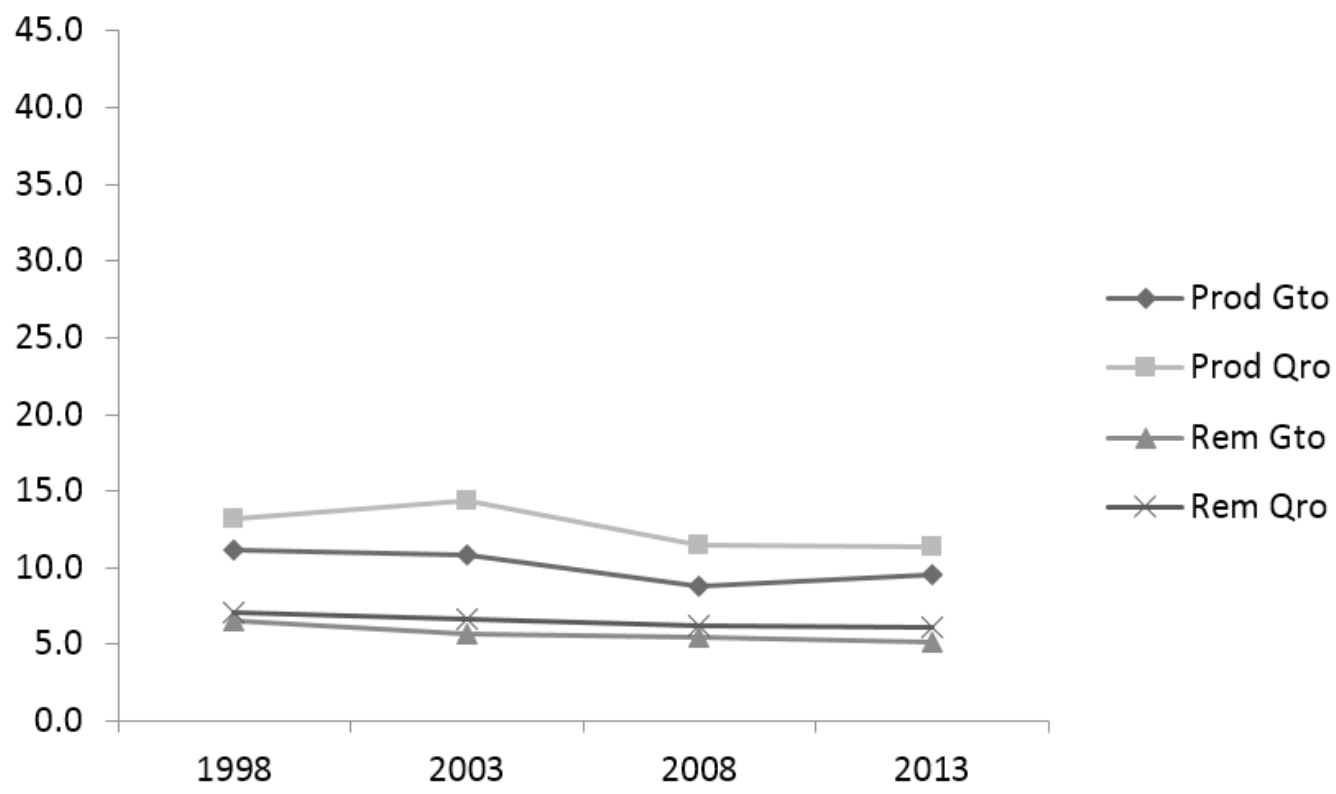

Fuente: Elaboración propia con datos de los censos económicos 1999, 2004, 2009 y 2014, INEGI.

\section{$\rightarrow$ 6. Conclusiones.}

El desarrollo de las regiones tiene diversas vertientes para su análisis. La variedad y riqueza de los estudios de índole mesoeconómica, basados en la observación de las iniciativas tecnológicas y de aprendizaje de los actores productivos, es sin duda una importante fuente de conocimiento del escenario regional. La perspectiva que nos brindan debe ser complementada con instrumentos que permitan conocer la estructura productiva básica y su evolución temporal, con lo cual es posible recoger la senda de desarrollo seguida. Bajo esta premisa hemos analizado con instrumentos estadísticos sectoriales a los estados de Guanajuato y Querétaro en un corte longitudinal de 15 años.

En ambos casos los servicios avanzados ocupan el lugar más dinámico, su estructura manufacturera es diversificada y han mantenido su papel de especialización nacional en sus mismos sectores. Son estados en que es importante la coevolución estadística entre la manufactura y los servicios avanzados, comparándolos con el nivel nacional.

Aunque no comparten algunas características como el tamaño de su producción y por tanto su aportación nacional, así como en la especialización manufacturera, ambos estados convergen en sus pautas de desarrollo básicas: su estructura sectorial, su diversificación manufacturera, sus productividades y remuneraciones sectoriales. En la coevolución entre manufactura y servicios también encontramos una convergencia en la mayor parte del período. 
Creemos que el ejercicio estadístico aplicado brinda bases para una mejor comprensión de la evolución de las dos entidades, que por las razones explicadas al inicio, son ejemplos de industrialización manufacturera de los últimos años. Hemos puesto la atención sobre el proceso conjunto de crecimiento de la manufactura y la terciarización, lo cual nos ha permitido mostrar la conformación de una región económica homogénea desde el punto de vista sectorial. 


\section{Anexo 1}

\section{Metodología}

Usamos el modelo de regresión lineal para calcular correlaciones simples y multivariadas, usando datos en series de tiempo o corte transversal, en niveles o logaritmos según la conveniencia de cada caso y de la información disponible para nuestros fines. La correlación indica la fuerza, dirección y proporcionalidad entre dos variables y dicho cálculo en su sentido estadístico, no implica alguna causalidad: el supuesto utilizado en este sentido en la presente metodología proviene de la literatura económica al respecto que indica que la manufactura tiene la capacidad de impulsar crecimiento en otros sectores.

\section{a. Índice de especialización.}

El índice de especialización es una medida tradicional que muestra la participación que un sub-sector tiene en un estado determinado, con relación a la participación de esa actividad a escala nacional. Un estado se especializará en un sub-sector si la aportación de éste, respecto al total del estado, es mayor que la aportación de ese sector a nivel nacional, es decir, si el valor del índice es mayor que la unidad. Si éste es menor que uno, indica que el estado no está especializado en dicho sub-sector. La especialización es mayor en la medida que rebasa la unidad.

El Índice de Especialización de cada rama lo calculamos con el valor agregado que reporta cada uno de los censos económicos que empleamos, y se define como:

$$
I E A=\frac{V A C B_{i j} * V A C B_{n}}{V A C B_{j} * V A C B_{i}}
$$

\section{Dónde}

$V A C B_{i j}$ : Valor agregado censal bruto rama (i) en el estado $\mathrm{j}$

$V A C B_{n}$ : Valor agregado censal bruto de la manufactura nacional

$V A C B_{j}$ : Valor Agregado censal bruto de la manufactura en el estado $\mathrm{j}$

$V A C B_{i}$ : Valor Agregado censal bruto de la rama (i) nacional 


\section{b. Índice de Herfindhal e Hirschman (IHH).}

La naturaleza de este indicador identifica y cuantifica en términos generales el comportamiento de cualquier estructura en algún momento del tiempo. Usada en Economía habitualmente para calcular el grado de concentración (y por analogía, de competencia) en determinados mercados, nosotros la empleamos para conocer la diversificación o concentración dentro del aparato manufacturero de cada estado.

En el ejercicio estadístico aquí desarrollado, no evaluamos la composición de un mercado en términos de competencia, si no la estructura del valor agregado del sector manufacturero de cada estado perteneciente a la muestra. El cien por ciento en el análisis de competencia en un mercado es el tamaño del mismo (medido en ventas totales, utilidades o alguna otra variable proxy); en el contexto de nuestra propuesta, el cien por ciento es la totalidad del Valor Agregado Censal Bruto (VACB) manufacturero estatal. Por otro lado, el equivalente a las empresas en el enfoque habitual, aquí son los 21 sub sectores que conforman al sector "31-33 industrias manufactureras" según la clasificación del Sistema de Clasificación Industrial de América del Norte (SCIAN), incluyendo al subsector "339 Otras industrias manufactureras".

Con datos de los Censos Económicos de los diferentes años usamos como base la participación porcentual de cada rama manufacturera para cada estado, por lo tanto el índice se calcula de la siguiente manera:

$$
I H H_{e}=\sum_{m=1}^{M} \% m^{2}
$$

\section{Donde}

$\mathrm{IHH}_{e}:$ Índice de Herfindahl e Hirschman del estado

m: rama manufacturera

\%: Participación de m en la producción total estatal

El IHH es la suma de los cuadrados de las participaciones de cada una de las m ramas manufactureras en la producción estatal de este sector; en el presente trabajo utilizamos como variable el Valor Agregado Censal Bruto, pues este indicador no toma en cuenta el consumo intermedio. Este índice toma valores que van desde cercanos a cero hasta 10,000. Entre más cercano es el resultado a dicho valor máximo, indica mayor concentración, por el contrario si el valor es más bajo, indica mayor diversificación de actividades, es decir, la producción más equilibrada. 


\section{c. Sensibilidad de los servicios ante incrementos en la producción manufacturera.}

Con la clasificación de servicios explicada en inciso 2 , calculamos el grado de respuesta de los diferentes tipos de servicios ante un incremento en mil pesos de la producción manufacturera; nos basamos en el modelo básico de regresión lineal con datos en niveles, tomando como muestra información estatal del Valor Agregado Censal Bruto (VACB) reportado en los Censos Económicos 1999, 2004, 2009 y 2014 por el Instituto Nacional de Estadística Geografía e Informática (INEGI) cada modelo se plantea de la siguiente manera:

$\mathrm{S}_{\mathrm{k}}=\beta_{0}+\beta_{1} * \operatorname{Man}+u_{t}$

En donde:

$S_{k}: V A C B$ del servicio $k$

Man: VACB manufactura total

$u_{t}:$ Termino estocástico

Al utilizar ambas series en niveles, el coeficiente $\beta 1$ expresa el cambio en el grupo de servicios cuando cambia la producción manufacturera expresada en valores alrededor de uno, dicha cifra la multiplicamos por mil, de esta manera sabemos en cuántos pesos se incrementa la producción de servicios ante un incremento de mil pesos en la manufactura total. Definimos este cambio como sensibilidad de los servicios ante el crecimiento en la producción de la manufactura.

Los datos de los que se compone la muestra para el cálculo de dicha sensibilidad en cada estado, son el valor agregado censal bruto de cada uno de sus municipios según la actividad evaluada. Para el caso del promedio nacional, la muestra son los valores estatales; es pertinente precisar que para éste último estadístico, se excluye al Distrito Federal pues presenta una dinámica distinta a la de la tendencia nacional. Esto queda ilustrado en la gráfica 11, en donde se muestra la relación que existe entre la producción manufacturera y de servicios (PIB) durante el periodo 2003-2013. Cada punto representa a los niveles de producción de ambos sectores de un estado en alguno de los diez años en cuestión. Es claro que el Distrito Federal, dentro del círculo punteado, lleva una lógica distinta al resto del país pues, sin reportar los niveles más altos de producción manufacturera, si presenta, y por mucho, los niveles más altos en producción de servicios, esto sugiere que su lógica de coevolución intersectorial responde de manera distinta o a otros factores que lo hacen no comparable al resto de los estados.

Para evitar el sesgo al alza que provoca un outlier como el que representa el Distrito Federal, optamos por hacer la evaluación prescindiendo de esta entidad. Por otro lado, constatamos que la tendencia en la sensibilidad general entre manufactura y servicios, no sólo es positiva sino de tipo cuadrática. 


\section{Gráfica 11}

Manufacturas y Servicios por estados 2003-2013

Miles de millones de pesos, precios constantes 2008

PIB Acts.

Terciarias

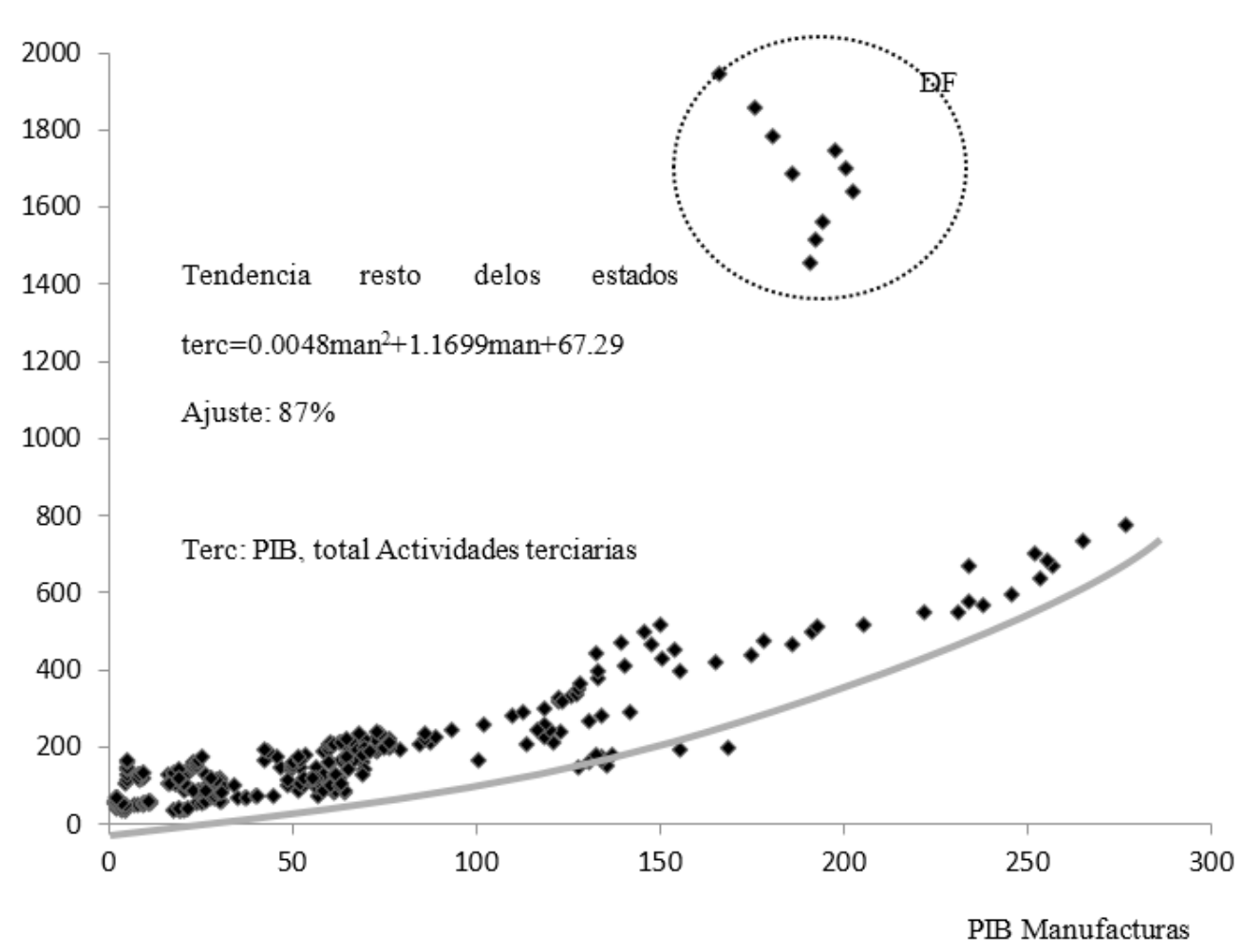

Fuente: Elaboración propia con datos del Banco de Información Económica BIE, INEGI. 


\section{Bibliografía y referencias documentales}

Álvarez, Lourdes , (2013). Las Redes de Innovación y el dinamismo de los sistemas productivos locales: El caso de Guanajuato, México. Chile, XXIX Congreso Latinoamericano de Sociología, ALAS Chile 2013, pp. 1-13.

Baró, Tomás, Ezequiel, (2013). ¿Desindustrialización o metamorfosis de la industria? La nueva relación entre las actividades manufacturera y terciaria. Economía Industrial, Volumen 387, pp. 33-47.

Bracamonte, Álvaro y Oscar, Contreras, (2011). Ciencia, tecnología e innovación para el desarrollo económico. Hermosillo, Sonora: El Colegio de Sonora, COECYT.

Carrillo, Jorge y María del Rosío, Barajas, (2007). Maquiladoras fronterizas. Evolución y heterogeneidad en los sectores electrónico y automotriz. Hermosillo, Sonora: El Colegio de la Frontera Norte, Miguel Angel Porrúa.

Casalet, Ravenna, Mónica, (2013). Actores y redes públicas y privadas en el desarrollo del sector aeroespacial internacional y nacional: el cluster de Querétaro, una oportunidad regional. En: La Industria Aeroespacial. México: FLACSO, México, pp. 93-134.

Casalet, Ravenna Mónica, (2000). El desarrollo de la capacidad innovadora de las empresas: El papel del ambiente en la formación y consolidación de las capacidades tecnológicas. México: FLACSO.

Contreras, Oscar y Paula, Isiordia, (2010). Local institutions, local networks and the upgrading challenge: Mobilizing regional assets to supply the global auto industry in Northern Mexico. International Journal of Automotive Technology and Management, 10(2-3), pp. 161-179.

Daniels, Peter y John, Bryson, (2002). Manufacturing Services and Servicing Manufacturing: Knowledge-based Cities and Changing Forms of Production. Urban Studies, 39(5-6), pp. 978-991..

Dutrénit, Gabriela, (2009). Sistemas regionales de innovación. Un espacio para el desarrollo de las Pymes. México: UAM, Textual.

Escalante, Roberto y Horacio, Catalán, (2011). El sector de manufacturas en Guanajuato y su participación en el mercado nacional. Problemas del Desarrollo, abril-junio, Issue 165 (42), pp. 87-112.

Estrada, Salvador, (2006). Diferencias regionales en la conducta tecnológica de las empresas manufactureras mexicanas: el caso de Guanajuato. Economía Sociedad y Territorio, V(20), pp. 821-869.

Greenfield, Harry, (1996). Manpower and the Growth of Poducer Services. Columbia: Columbia University Press, New York.

Hansen, Niles, (1990). Do producer services induce regional economic development ?. Journal of Regional Science, 30(4), pp. 465-476.

Hualde, Alfredo, (2010). Pymes y sistemas regionales de innovación: la industria del software en Baja California y Jalisco. México: El Colegio de la Frontera Norte, UAM, Textual.

INEGI, Banco de Información Económica.

INEGI, Censos Económicos varios años

Jasso, Villazul, Javier y Lourdes, Marquina, Sánchez, (2014). LA SERVIMANUFACTURA: UN CONCEPTO PARA ILUSTRAR. Universidad Autónoma de Baja California, s.n., disponible en http://docplayer. es/3480608-Universidad-autonoma-de-baja-california-facultad-de-contaduria-y-administracion-academia-de-ciencias-administrativas-a-c-coordinacion-de-edicion.html

Kaldor, Nicholas, (1966). Causes of the Slow Rate of Economic Growth of the United Kingdom: an Inaugural Lecture. London: Cambridge University Press.

Lara, Arturo, (2007). Co-evolución de empresas, maquiladoras, instituciones y regiones: una nueva interpretación. México: Editorial Porrúa.

Maillat, Denis y Antoine, Bailly, (1989). Servicios a las empresas y desarrollo regional. Ekonomiaz, Issue 13-14, pp. 128-137. 
Martínez, Adriana, (2015). Sistema estatal de innovación y la importancia de los factores institucionales. El caso de Guanajuato. En: Experiencias estatales y transfronterizas de innovación en México. Tijuana, Baja California: El Colegio de la Frontera Norte, COMECSO, pp. 99-136.

Martínez, Adriana, Alejandro, García, y Eunice Taboada, (2012). Análisis de instrumentos de política de innovación: Trayectoria de 16 años del Consejo de Ciencia y Tecnología de Guanajuato. En: Dilemas de ka Innovación en México. Tijuana, Baja California: El Colegio de la Frontera Norte, pp. 337-410.

Martínez, Adriana, (2009). El Sistema Sectorial de Innovación de Calzado: el caso Guanajuato. En: Sistemas de Innovación en México. Regiones, redes y sectores. México: Plaza y Valdez, pp. 59-88.

Micheli, Jordy, (2014). Desarrollo regional en México durante 2003-2011: Polarización de la manufactura y diversificación de los servicios. En: Tecnología y competitividad: conceptos, experiencias y prácticas. México: El Colegio de Sonora, pp. 55-78.

Micheli, Jordy y Eduardo Valle, (2016). Los servicios avanzados y la nueva geografía de la industria automotriz en México. Una propuesta para evaluar el desarrollo local en el período 1998-2013. En Martínez , Adriana y Jorge Carrillo (coord.) : Innovación, redes de colaboración y sostenibilidad. Experiencias regionales y tendencias internacionales de la industria automotriz.. México: Am Editores / ENES León /UNAM/ Colson / CIAD.

Pilat, Dirk y Anita, Wölfl, (2005). Measuring the Interaction Between Manufacturing and Services. OECD Science, Technology and Industry Working Papers.

Rózga, Ryszard, (2010). Estudios territoriales: modelos territoriales de innovación; su reflejo y aplicación en México. En: Innovación ante la sociedad del conocimiento. México: Facultad de Economía, UNAM, Plaza y Valdes Editores, pp. 157-177.

Villavicencio, Daniel, Juana Hernández y Leonardo Souza, (2013). Capacidades y oportunidades para el desarrollo de la industria aeronáutica en Querétaro. En: La industria Aeroespacial. México: FLACSO, México, pp. 49-91.

Villavicencio, Daniel y Pedro, López de Alba, (2009). Sistemas de Innovación en México regiones, redes y sectores. México: CONACYT/Plaza y Valdés.

Villavicencio, Daniel, Adriana, Martínez, y Pedro, López de Alba, (2011). Dinámicas institucionales y políticas de innovación en México. México: UAM Xochimilco, CONACYT, CONCITG, Plaza y Valdés Editores. 


\section{Números anteriores:}

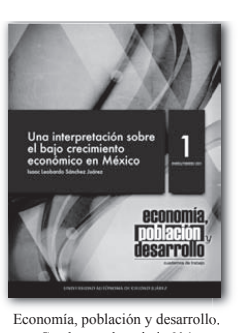

Economía, población y desarrollo
Cuadernos de trabajo №1

Enero-Febrero 2011

Una interpretación sobre el bajo
crecimiento económico en Méxice

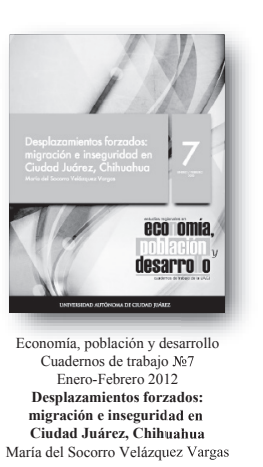

Ciudad Juárez, Chihuahua
Maria del Socorro Veläquez Vargas

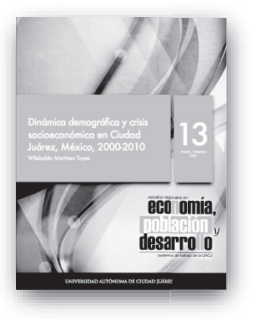

Economía, población y desarrollo
Cuarednos de trabajo № 13 ńmica demogŕfica y

fica y crisis

México, 2000-2010
Wilebaldo Martinez Toyes
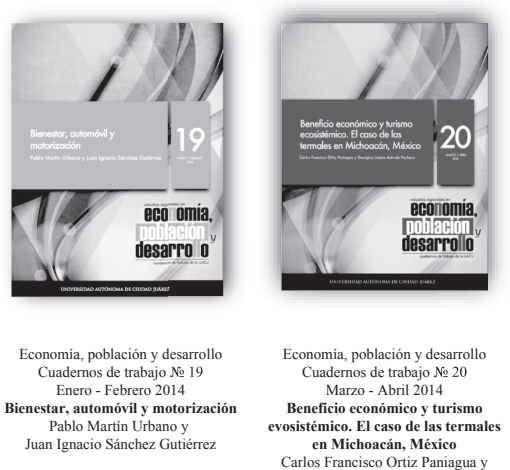
Carlos Francisco Ortizi Paniagua y
Georgina Jatzire Arévalo Pacheco
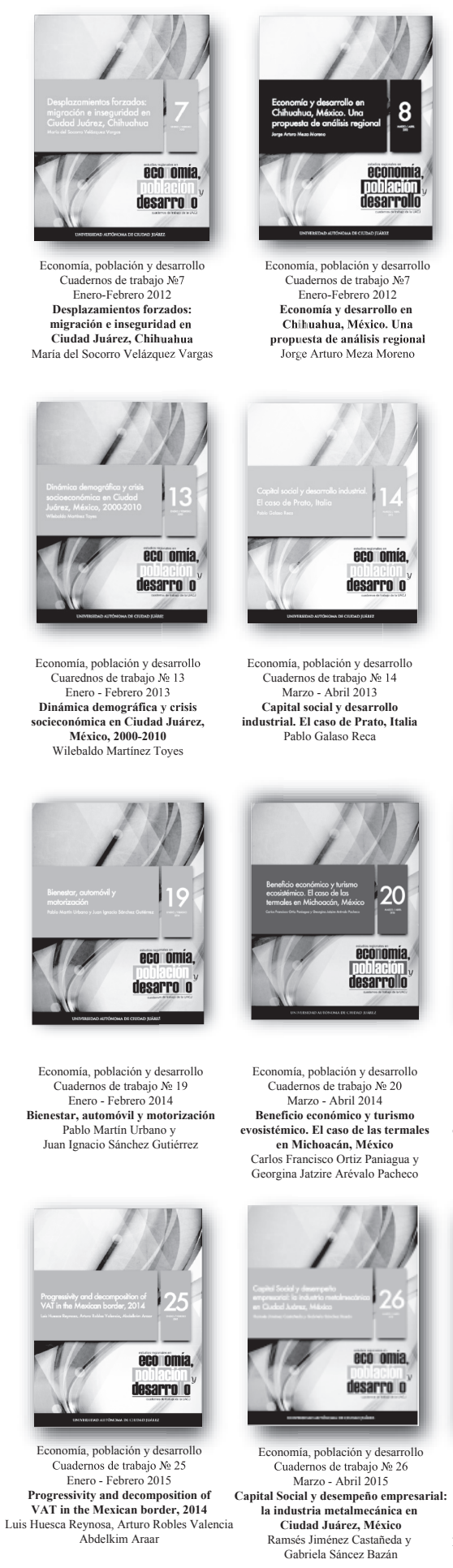

Economía, población y desarrollo
Cuadernos de trabajo № 14

Capital social y desarrollo

strial. El caso de Prato, It

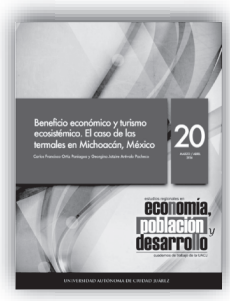

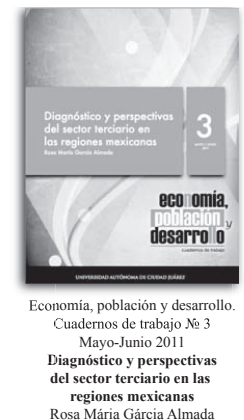
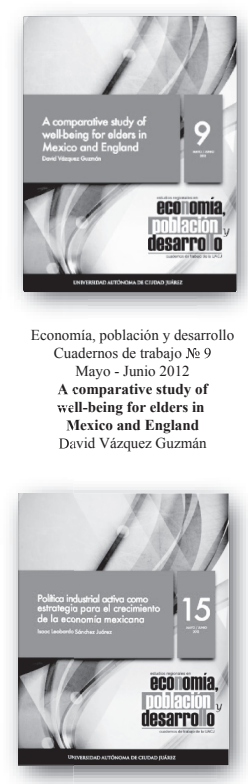

Economía, población y desarrollo
Cuadernos de trabajo № 15

Política industrial activa como de la economia mexicana
Isaac Leobardo Sánchez Juárez
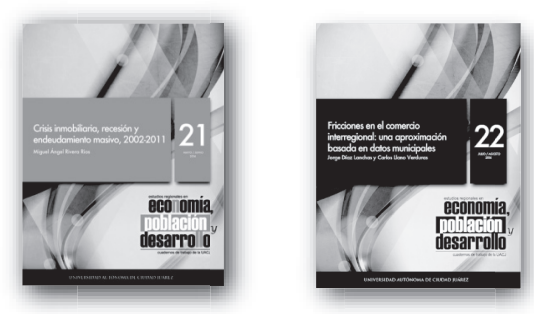

Economía, población y desarrol
Cuadernos de trabajo № 21 Mayo - Junio 2014 Crisis inmobiliaria, recesión y
endeudamiento masivo, 2002 -2011 Miguel Ángel Rivera Rios
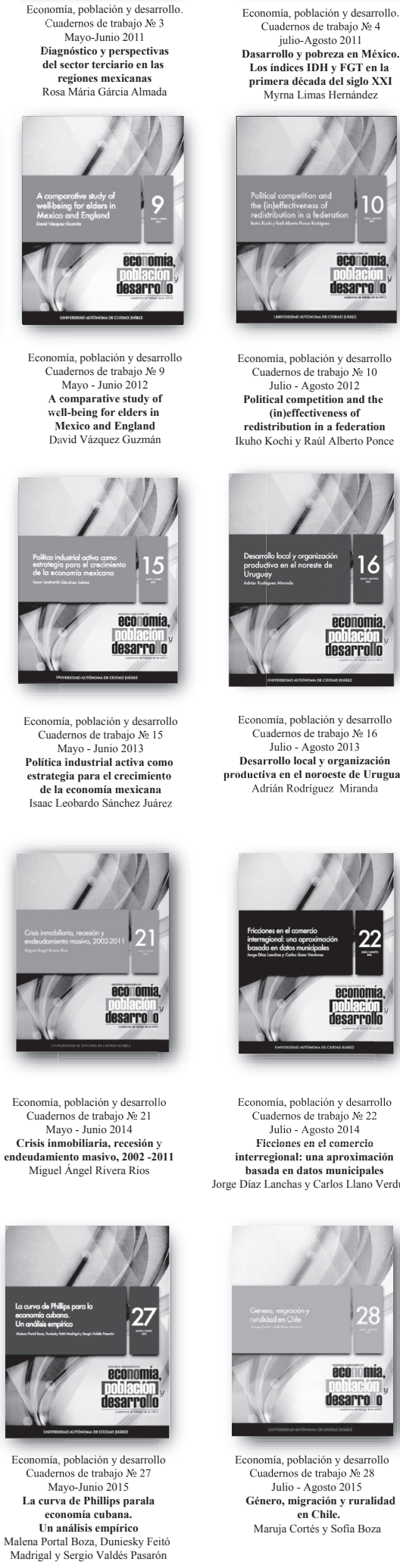

julio-Agosto 2011
rollo y pobreza en

Dasarrollo y pobreza en México.
Los indices IDH y FGT en la primera década del siglo XXI

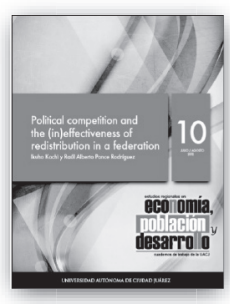

Economía, población y desarrollo Cuadernos de trabajo № 10
Julio - Agosto 2012 Political competition and the redistribution in a federation
lkuho Kochi y Raúl Alberto Ponce

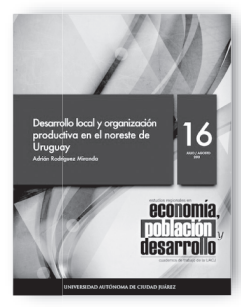

Economía, población y desarrollo
Cuadernos de trabajo № 16

Desarrollo local y organización
productiva en el noroeste de Urugua
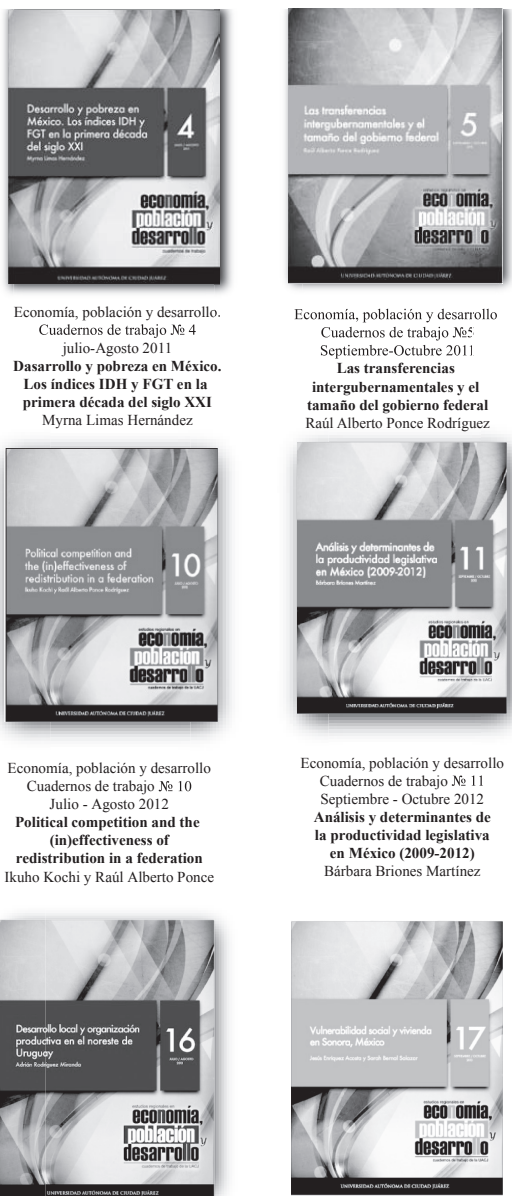

Adrián Rodriguez Miranda

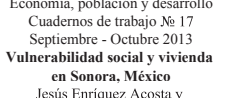
Jesús Enríquez Acosta y
Sarah Bernal Salazar

conomía, población y desarrollo
Cuadernos de trabajo №5
Septiembre-Octubre 2011

Septiembre-Octubre 205
Las transferencias

intergubernamentales $\mathrm{y}$ el
tamaño del gobierno federal

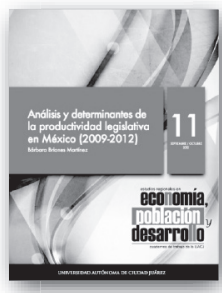

Economía, población y desarrollo

Cuadernos de trabajo № 11

Análisis y determinantes de en México (2009-2012)
entis

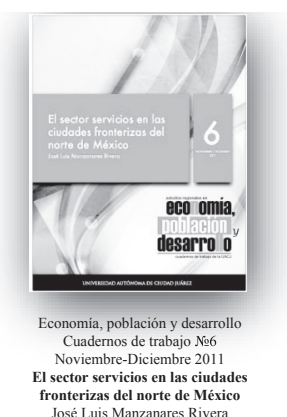

José Luis Manzanares Rivera

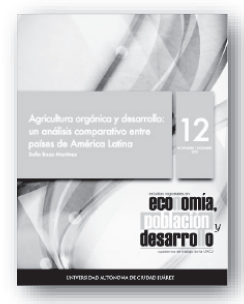

Economía, población y desarrollo Noviembre - Diciembre 2012

un analisis comparativo entre

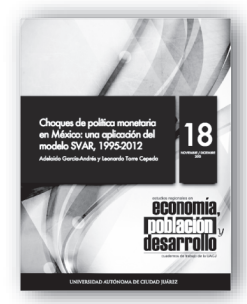

Cunomia, población y desarrollo Cuadernos de trabajo № 18

Choques de politica monetaria en México: una aplicación del
modelo SVAR, 1995-2012
Adelaido García-Andés y Leonardo Torre Ce
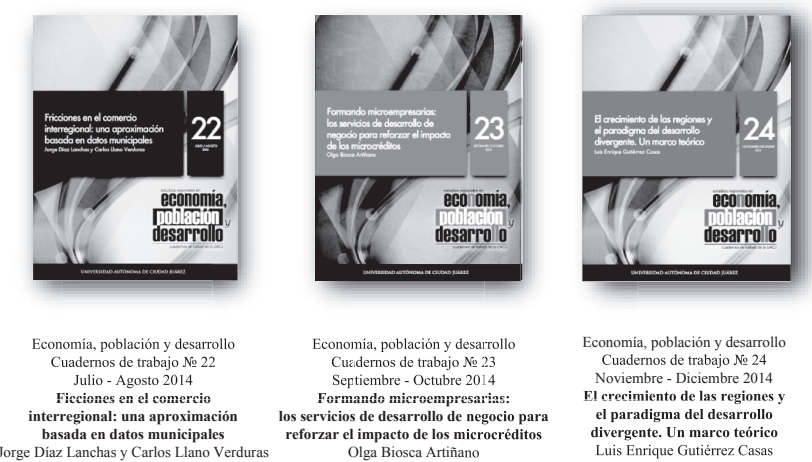

Cuadernos de Noviembre - Diciembre 2014 El crecimiento de las regiones $y$ el paradigma del desarrollo
divergente. Un marco teórico Luis Enrique Gutiérrez Casas
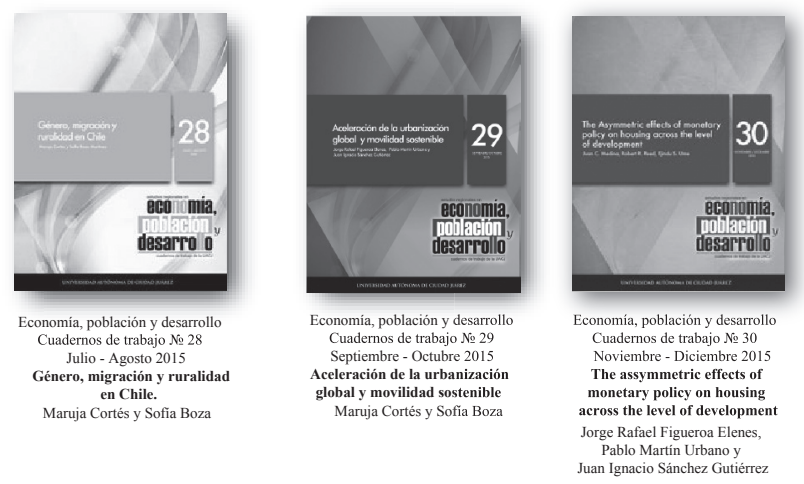
Números anteriores:

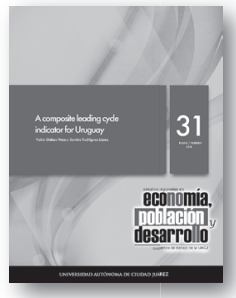

Economia, población y desarrollo Uadernos de trabajo № 31 Enero - Febrero 2016 indicator for Uruguay Pablo Galaso Reca y Sandra Rodriguez López

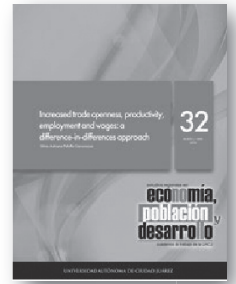

Economia, población y desarrollo Cuadernos de trabajo № 32
Marzo - Abril 2016

Increased trade openness, productivity, a difference-in-differences approac Silvia Adriana Peluffo Geronazzo

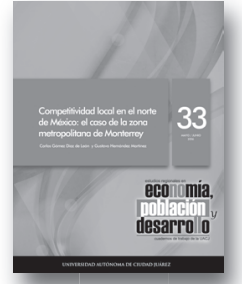

Economia, población y desarrollo Cuadernos de trabajo № 33

Competitividad local en el norte
de México: el caso de la zona

metropolitana de Monterrey

Carlos Gómez Díaz de León y

Gustavo Hernández Martinez

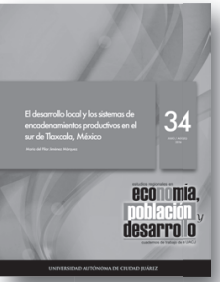

Economia, población y desarrollo

Cuadernos de trabajo № 34
Julio - Agosto 2016

El desarrollo local y los

promas de encadenamientos

Poductivos en el sur de
Tlaxcala, México

Tlaxcala, México
Maria del Pilar Jiménez Márquez

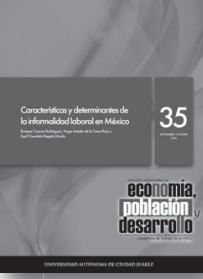

Economia, población y desarrollo Cuadernos de trabajo № 35

Características y determinantes

de la informalidad laboral

en México

Enrique Cuevas Rodríguez,
Hugo Antolin de la Torre Ruiz 


\section{$\rightarrow$ Normas Editoriales}

\section{Para el documento general:}

Tipo de letra: Times New Roman.

Tamaño: 11 puntos.

Interlineado: 1.5 espacios.

Títulos y subtítulos:

El texto principal en 11 puntos. Títulos 12 puntos (en resaltado). Subtítulos 11 puntos. Cada título y subtítulo deberá numerarse bajo el siguiente orden: $1,1.1,2,2.1,2.2 \ldots$

La extensión máxima de los cuadernos de trabajo será de 40 cuartillas.

La primera vez que se emplee una sigla en el texto se especificará primero su equivalencia completa y después la sigla.

\section{Hoja de presentación:}

Título:

14 puntos, centrado, resaltado.

Nombre de autor(es):

12 puntos

Resumen y abstract:

Debe incluir resumen en español y abstract (diez puntos), no mayor a 250 palabras

Palabras clave:

Incluir entre tres y cinco palabras clave, en español e inglés

Referencia del autor o autores:

Institución de adscripción, grado académico y líneas-grupos de investigación que desarrolla y a los que pertenece.

\section{Sistema de referencia de citas:}

Harvard-APA

Las citas bibliográficas en el texto deberán incluir entre paréntesis sólo el apellido del autor, la fecha de publicación y el número de página; por ejemplo: (Quilodrán, 2001: 33).

\section{Notación en sección de bibliografía y fuentes de información:}

Se deberá incluir al final del texto. Toda referencia deberá estar mencionada en el texto o notas de pie de página.

Cada referencia iniciará con el primer apellido o los apellidos, luego el nombre del autor, y después, entre paréntesis, el año de publicación seguido de un punto. Ejemplos:

Se deberá incluir al final del texto. Toda referencia deberá estar mencionada en el texto o notas de pie de página.

Cada referencia iniciará con el primer apellido o los apellidos, luego el nombre del autor, y después, entre paréntesis, el año de publicación seguido de un punto. Ejemplos:

Artículo:

Ros, Jaime (2008). "La desaceleración del crecimiento económico en México desde 1982”, en Trimestre Económico, vol. 75, núm. 299, pp. 537-560.

Libro:

Villarreal, René (2005). Industrialización, competitividad y desequilibrio externo en México. 
Un enfoque macroindustrial y financiero (1929-2010), México, Fondo de Cultura Económica. Capítulo de libro:

Castillo, Manuel Ángel (2003). "La política de inmigración en México: un breve recuento", en Manuel Ángel Castillo, Alfredo Lattes y Jorge Santibáñez (coords.), Migración y fronteras, Tijuana, El Colegio de la Frontera Norte / Asociación Latinoamericana de Sociología / El Colegio de México, pp. 425-451.

\section{Notas de pie de página:}

Se utilizarán para hacer indicaciones complementarias, aclaraciones o ampliación de una explicación. La notas de pie de página en Times New Roman, 10 puntos.

\section{Tipología de imágenes dentro del texto:}

Cuadro

Gráfica

Diagrama

Mapa

Figura

Todas las imágenes deben ser numeradas y mencionadas dentro del texto. A toda imagen debe incluirse la fuente.

Las indicaciones de la imagen: tipo y número de imagen, título de imagen y fuente se escriben en 10 puntos. En el texto poner como imagen los mapas, figuras, gráficas y diagramas -con el ánimo de no perder el formato realizado por el autor.

\section{Ecuaciones y fórmulas:}

Si se utilizan ecuaciones o fórmulas deberá utilizarse el editor de ecuaciones de Word y numerarse.

\section{Envío de trabajos}

Los trabajos deben ser enviados a la dirección de correo: lgtz@uacj.mx. Con el Dr. Luis Enrique Gutierrez Casas, editor de esta publicación.

La aceptación de cada colaboración dependerá de la evaluación de dos dictaminadores especialistas en la materia que se conservarán en el anonimato, al igual que el autor (autores) para efectos de la misma. 


\section{$\rightarrow$ Editorial Guidelines}

\section{For General Document:}

Font type: Times New Roman.

Size: font size 11 .

Paragraph: 1.5 line spacing.

Titles and subtitles: Main text font size 11. Titles font size 12 (Bold). Subtitles font size 11.

Each title and subtitle should be numbered in the following order: 1, 1.1, 2, 2.1, 2.2...

The maximum length of the workbooks will be 40 pages.

The first time an abbreviation is used in the text will be specified first complete equivalence and then stands.

\section{Front cover:}

Title:

Font size 14, centered, Bold.

Author name(s):

Font size 12.

Abstract:

It should include abstract in Spanish and abstract (font size 10), no more than 250 words.

Keywords:

Include three to five keywords, in Spanish and English.

Reference of author:

Institution of affiliation, academic degree and line-developed by research groups and belonging.

\section{Bibliographical appointment system:}

Harvard-APA

Citations in the text should include between parentheses only the author's name, publication date and page number, for example:

(Quilodrán, 2001: 33).

\section{Notation about Bibliography section and Information fonts:}

Should be included at the end of the text. All references must be mentioned in the text or footnotes page.

Each reference starts with the first name or last name, then the name of the author, and then, in parentheses, the year of publication followed by a period. Examples:

Article:

Ros, Jaime (2008). “La desaceleración del crecimiento económico en México desde 1982”, en Trimestre Económico, vol. 75, núm. 299, pp. 537-560.

Book:

Villarreal, René (2005). Industrialización, competitividad y desequilibrio externo en México. Un enfoque macroindustrial y financiero (1929-2010), México, Fondo de Cultura Económica.

Book chapter:

Castillo, Manuel Ángel (2003). "La política de inmigración en México: un breve recuento”, en Manuel Ángel Castillo, Alfredo Lattes y Jorge Santibáñez (coords.), Migración y fronteras, Tijuana, E1 Colegio de la Frontera Norte / Asociación Latinoamericana de Sociología / El Colegio de México, pp. 425-451. 


\section{Footnotes:}

Must be used to make additional indications, clarification or expansion of an explanation. The footnotes must be in Times New Roman, font size 10.

\section{Image typology inside text:}

Picture

Graph

Diagram

Map

Figure

All images must be numbered and mentioned in the text, should include the source image. The indications of the image: type and number of image, image title and source are written in 10 font size. In the text set as image maps, figures, graphs and charts-with the intention of not losing the formatting by the author.

\section{Equations and Formulae:}

When using equations or formulas should be used in Microsoft Word equation editor and numbered.

\section{Paper sending}

Entries must be sent to the email address: lgtz@uacj.mx. With Dr. Luis Enrique Gutiérrez Casas, editor of this publication.

Acceptance of each collaboration will depend on the evaluation of two examiners skilled in the art to be kept anonymous, like the author(s) for the same purposes. 


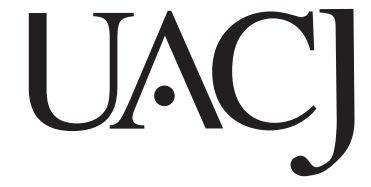

Esta obra se terminó de imprimir en octubre de 2016 Cd. Juárez, Chihuahua, México.

Tiraje: 120 ejemplares 
Cuadernos de Trabajo de la UACJ, Universidad Autónoma de Ciudad Juárez, número 36, noviembre-diciembre de 2016

\section{Director y editor}

Dr. Luis Enrique Gutiérrez Casas

\section{Comité editorial}

Sección internacional

Dra. Sofía Boza Martínez (Universidad de Chile, Chile)

Dra. Olga Biosca Artiñano (Glasgow Caledonian University, Reino Unido)

Dra. Ángeles Sánchez Díez (Universidad Autónoma de Madrid, España)

Dr. Thomas Fullerton Mankin (University of Texas at El Paso, Estados Unidos)

Dr. Adrián Rodríguez Miranda (Universidad de la República, Uruguay)

\section{Sección local}

(Universidad Autónoma de Ciudad Juárez)

Dra. Myrna Limas Hernández

Dra. Ikuho Kochi

Dr. Raúl Alberto Ponce Rodríguez

Dr. Isaac Leobardo Sánchez Juárez

Dr. Héctor Alonso Barajas Bustillos 